\title{
Firefly Algorithm in Biomedical and Health Care: Advances, Issues and Challenges
}

\author{
Janmenjoy Nayak ${ }^{1} \cdot$ Bighnaraj Naik ${ }^{2} \odot \cdot$ Paidi Dinesh $^{3} \cdot$ Kanithi Vakula ${ }^{3} \cdot$ Pandit Byomakesha Dash ${ }^{2}$
}

Received: 21 May 2020 / Accepted: 5 September 2020 / Published online: 26 September 2020

(c) Springer Nature Singapore Pte Ltd 2020

\begin{abstract}
Since the past decades, most of the nature inspired optimization algorithms (NIOA) have been developed and become admired due to their effectiveness for resolving a variety of complex problems of dissimilar domain. Firefly algorithm (FA) is well-known, yet efficient nature inspired swarm intelligence (SI) based metaheuristic algorithm. Since from its initiation, FA has become well-liked between the researchers due to its competence and turn out to be an interesting technique for the practitioners as well as researchers for solving the problems of numerous fields of research such as classifications, clustering, neural networks, biomedical engineering, healthcare as well as other research domain. Moreover, there is an outstanding track record of FA in solving biomedical engineering (BME) and healthcare (HC) problems. Abundant complexities have been worked out with the assist of FA and its variants. By taking these particulars into concern, in this paper, a first ever in-depth analysis has been addressed on the variants, importance, applications as well as enhancements of FA in BME as well as HC. The major intention behind this investigative work is to motivate the researchers to improve and innovate new solutions for multifaceted problems of healthcare and biomedical engineering using FA.
\end{abstract}

Keywords Firefly algorithm - Swarm intelligence $\cdot$ Biomedical engineering and healthcare $\cdot$ Nature-inspired algorithm . Metaheuristics

I on behalf of the authors would like to state that the above manuscript is our original research work and it has not been published elsewhere. Also, it has not been submitted to any journal for publication.

\footnotetext{
Bighnaraj Naik

bnaik_mca@vssut.ac.in

Janmenjoy Nayak

jnayak.cse@adityatekkali.edu.in

Paidi Dinesh

dinesh.pydi98@gmail.com

Kanithi Vakula

vakku.bi@gmail.com

Pandit Byomakesha Dash

byomakeshdash2000@gmail.com

1 Department of Computer Science and Engineering, Aditya Institute of Technology and Management (AITAM), K Kotturu, Tekkali 532201, Andhra Pradesh, India

2 Department of Computer Application, Veer Surendra Sai University of Technology, Burla 768018, Odisha, India

3 Department of Computer Science and Engineering, Sri Sivani College of Engineering, Srikakulam 532402, Andhra Pradesh, India
}

$\begin{array}{ll}\text { Abbreviations } & \\ \text { ABC } & \text { Artificial Bee Colony } \\ \text { AFA } & \text { Adaptive Firefly Algorithm } \\ \text { AIP } & \text { Analogue image processing } \\ \text { ANN } & \text { Artificial Neural Network } \\ \text { APSO } & \text { Accelerated Particle Swarm } \\ & \text { Optimization } \\ \text { BCFCM } & \text { Bias Corrected Fuzzy C-Means } \\ \text { BF } & \text { Bilateral Filter } \\ \text { BFA } & \text { Binary Firefly Algorithm } \\ \text { BME } & \text { Bio Medical Engineering } \\ \text { BPSO } & \text { Binary Particle Swarm Optimization } \\ \text { BSP } & \text { Biomedical Signal Processing } \\ \text { CDSS } & \text { Clinical Decision Support System } \\ \text { CFA } & \text { Chaotic Firefly Algorithm } \\ \text { CGA } & \text { Controlled Genetic Algorithm } \\ \text { CM } & \text { Chinese Medicine } \\ \text { CMFA } & \text { Compact FA } \\ \text { CMS } & \text { Chaotic Maps } \\ \text { CNN } & \text { Convolutional based Neural Network } \\ \text { CSA } & \text { Cuckoo Search Algorithm } \\ \text { CT } & \text { Computer Tomography } \\ \text { CV } & \text { Cross Validation }\end{array}$




\begin{tabular}{|c|c|c|c|}
\hline $\mathrm{DE}$ & Differential Evolution & MFF & Multi Features Fusion \\
\hline DF & Dragonfly & MFF & Modified Firefly \\
\hline DFA & Discrete Firefly algorithm & MIF & Myocardial Infarction \\
\hline DFA-RF & Discrete firefly based Random Forests & MI & Medical imaging \\
\hline \multirow[t]{2}{*}{ DFA-SVM } & Discrete firefly based Support Vector & MLPNN & Multilayered Perceptron Neural Network \\
\hline & Machines & MMG & Magnetomyography \\
\hline \multirow[t]{2}{*}{ DHAFA } & Dynamic hybrid adaptive firefly & MNN & Modular Neural Network \\
\hline & algorithm & MOFA & Moth FA \\
\hline DIP & Digital image processing & MR & Magnetic Resonance \\
\hline DNN & Deep Neural Network Model & MRI & Magnetic Resonance Imaging \\
\hline DR & Diabetic retinopathy & MSE & Mean square error \\
\hline DWT & Discrete wavelet transforms & MVC-NMF & Minimum Volume Constrained Non- \\
\hline EA & Evolutionary algorithms & & negative Matrix Factorization \\
\hline ECG & Electro-Cardiogram & NCHT & Natural ordered complex Hadamard \\
\hline $\mathrm{EE}$ & Extort end-members & & transform \\
\hline EEG & Electro Encephalo Gram & NF & Neuro-fuzy \\
\hline EFA & Enhanced FA & NFN & Neuro-fuzy network \\
\hline ELM & Extreme learning model & NIA & Nature Inspired Algorithms \\
\hline $\mathrm{ES}$ & Evolution Strategies & $\mathrm{NIO}$ & Nature Inspired optimization \\
\hline FA & Firefly Algorithm & NIOA & Nature Inspired Optimization Algorithm \\
\hline FCM & Fuzzy C-Means & OAEI & Ontology alignment evaluation initiative \\
\hline \multirow[t]{2}{*}{ FFABPNN } & Fused Feature Adaptive Firefly Back & OCR & Optimum compression ratio \\
\hline & Propagation Neural Network & OFA & Opposition based FA \\
\hline FFNN & Feed Forward Neural Network & PCA & Principal component analysis \\
\hline FF-SVM & FireFly-based Support Vector Machine & PP & Post processor \\
\hline FNB & Fine needle biopsy & PSFA & Particle swarm firefly algorithm \\
\hline GA & Genetic algorithm & PSN & Peak signal to noise \\
\hline GTI & Ground-Truth-Image & PSNR & Peak Signal to Noise Ratio \\
\hline GWO & Grey-wolf optimizer & PSO & Particle Swarm Optimization \\
\hline HAS & Harmony Search & PSVM & Penalized Support Vector Machine \\
\hline HBMO & Honey Bee Mate Optimization & RDFG_CDQO & Restricted divergence based stochastic \\
\hline $\mathrm{HC}$ & Health Care & & clinical big data query Optimizer \\
\hline HFA & Hybridized Firefly Algorithm & RMSE & Root mean square error \\
\hline HT & Hadamard Transform & RS-CDQO & Restricted Selection Based clinical big \\
\hline \multirow[t]{2}{*}{ HTRSFA } & Hybrid Tolerance Rough Set-Firefly & & data query Optimizer \\
\hline & Algorithm & SAM & Simulated Annealing Method \\
\hline IC & Image classification & SCAD & Smoothly Clipped Absolute Deviation \\
\hline ICP & Iterative Closest Point & SDFA & Simple Discrete FA \\
\hline ID & Image detection & SE & Shannon Entropy \\
\hline IFCM & Intuitionistic Fuzzy C-Means & SFC & Supervised fuzzy clustering \\
\hline \multirow[t]{2}{*}{ IFCM-FA } & Intuitionistic Fuzzy C-Means based Fire- & SI & Swarm Intelligence \\
\hline & fly Algorithm & SMACC & Sequential maximum angle convex cone \\
\hline IP & Image processing & SNR & Signal-to-Noise Ratio \\
\hline IPP & Image pre-processing & SP & Signal processing \\
\hline IR & Image registration & SSI & Structural similarity index \\
\hline IS & Image segmentation & STRSPSO-QR & Supervised Tolerance Rough Set-PSO \\
\hline JE & Joint entropy & & based Quick Reduct \\
\hline LFFA & Levy Flight FA & STRSPSO-RR & Supervised Tolerance Rough Set-PSO \\
\hline LLS & Linear Least-Squares & & based Relative Reduct \\
\hline LLWNN & Local Linear Wavelet Neural Network & SVM & Support Vector Machine \\
\hline MA & Mimetic Algorithm & SVMC & Support Vector Machine Classifier \\
\hline MEG & Magnetoencephalography & TRS & Tolerant Rough Set \\
\hline MFA & Modified Firefly Algorithm & & \\
\hline
\end{tabular}




$\begin{array}{ll}\text { TRSFQR } & \begin{array}{l}\text { Tolerance Rough Set Firefly based Quick } \\ \text { Redacts }\end{array} \\ \text { VCA } & \text { Vertex Component Analysis } \\ \text { VRMSE } & \text { Vector root mean square error } \\ \text { VSSFA } & \text { Variable step size firefly algorithm }\end{array}$

\section{Introduction}

Nature inspired optimization (NIO) is the reflection of the development of computational methods through activities or behaviors of several natural creatures/plants/elements. These computing methods led to the advancement of algorithms known as Nature Inspired Algorithms (NIA). The literature of NIO has been grown significantly over last one decade. The major concern of emerging such type of algorithms is to escalate engineering problems. As the entire world is poignant to industrialization, engineering problems are becoming more intricate and complicated to optimize. This is due to the increasing dimensions, dealing with time and space complexity and handling complex variables. To deal such condition, NIA are intended to optimize multi-objective functions and resolve problems of NP-hard for larger dimensions, variables, etc. NIA are mostly classified into Evolutionary Algorithms (EA) and Swarm Intelligence (SI) based algorithms (including some of the other algorithms based on chemical and physical properties). EA are completely depending on evolutionary actions of natural systems. To optimize the difficult problems, EA utilize recombination as well as mutation operators. Differential Evolution (DE) [1] and Genetic algorithm [2] are the two most successful evolutionary algorithms. On the other hand, SI based algorithms are also known as swarm optimization methods, used to optimize specific problems by imitating the combined actions of natural swarms. The phrase swarm is utilized for a collection of existing natural beings such as birds, fishes, and insects like bees, termites, and ants, etc. The constituents in the swarm interrelate with one another by not including any central command and they move through the data acquired from their vicinity [3]. Ant colony [4], particle swarm optimization [5], multi-swarm PSO [6] cuckoo search [7] and firefly algorithms [8] etc are some examples of SI algorithm, where perceptions like joint activities and the combined swarming association of organisms regularly guide to the SI. However, enhanced searching methods over difficult exploration spaces can be attained by representatives that are following local rules devoid of every central control [9].

When we focus on successful SI based algorithms, FA is population-based and is on the hot spot. If our importance is based on communication of unusual agents, algorithms can be classified as attract or non-attract based. FA is the good example of attract based algorithms, replicated by nature. These days FA has turned out to be a significant element of
SI which is applied in several areas of engineering and optimization problems. Xin-She Yang [8] has introduced FA in 2007. The algorithm was inspired by imitating the flashing activities of fireflies for the purpose of food gaining. The attraction of each firefly towards one another is signified by their intensity, which raises or reduces depending on distances among the flies. The brightness of individual firefly is contrasted with other. High light intensity flies attracts the flies with low light intensity, thereby reducing the distance and modernizing its own intensity. The firefly with elevated brightness (intensity) and slightest distance is the most excellent solution of an objective function. Firefly algorithm is applied in many applications such as least computation time for digital image compression [10,11], feature selection [12], antenna design optimization [13], multi objective load dispatch problems $[14,15]$, scheduling and travelling salesman problem [16, 17], classifications, clustering [18], neural networks [19], optimal divergence based electromagnetic \& fuzzy devise for multi-objective UCP $[20,21]$ as well as BME [22], HC [23, 24] etc.

Along with the developments in the updation of background knowledge, important evolution has been made in the effectiveness and number of automated methods that are being used in medical studies [22], where huge amount of information will be accumulated in medical databases. Health care systems produce enormous amount of data and it is gathered in medical databases and the physical categorization of this data becoming very complicated. Therefore, there is a rising attention in increasing automated techniques for medical data analysis [25]. In this paper, the main goal is to emphasize on the firefly algorithm and its significance in biomedical engineering and healthcare. Biomedical engineering is a control that proceeds information in engineering, medicine as well as biology, and advances human health through the actions of cross-discipline that incorporate the engineering sciences through the clinical practice and biomedical sciences. Healthcare is the preservation or development of health by means of anticipation, diagnosis, and handling of disease, injury, illness and other physical and mental impairments in people. Firefly algorithm has been utilized in important medical fields such as breast cancer classification, human retinal images, breast tumor classification, detection of brain tumor, health monitoring etc. Also, assortment of soft computing methods such as evolutionary algorithms, fuzzy logic, swarm-based algorithms and neural networks is obtained to take enhanced implementation of BME \& HC. However, FA has always been lingered an excellent choice for solving biomedical as well as healthcare problem due to its high flexibility in producing optimal solutions and effectiveness. This paper addresses the appliance of FA in these two major engineering problems such as biomedical and healthcare in a greater aspect. Moreover, the variants of FA and their significance in the above two applications areas are 
highlighted along with the concern towards the issues and future challenges of FA.

Despite of the above some disadvantages of meta-heuristic algorithms, the classical or standard Firefly Algorithm have some essential characteristics which make it successful and efficient such as:

1. This algorithm has local attraction responsiveness, better than long distance attraction. By incorporating this, FA divides the population into subgroups which makes it efficient and suitable for non-linear and multi-model optimization problem.

2. FA does not take decisions by using historic individual best and explicit global best, thereby overcome the drawback of premature convergence.

3. FA has good control over the scaling parameters which makes it self-adaptable.

The main contribution of this study is to: (1) effective analysis on the applicability of FA and its variations in BM \& HC, (2) analyze the appropriateness of FA in diverse domains of BM \& HC, (3) demonstrate the effectiveness of FA for solving $\mathrm{BM} \& \mathrm{HC}$ related issues with several performance factors and comparisons, (4) critically investigate the quantitative factors such as performance, publications and domain specific applications of FA in several research dimensions of BM \& HC, (5) qualitative study on various issues and factors involved in the success and failure of FA in BM \& HC with some future unexplored areas. The remaining part of the paper is represented as follows. Section "Firefly Algorithm" illustrates about the structure and working concepts of firefly algorithm. Section "Systematic Literature Review Process" depicts the systematic literature review process considered for this study. The variants of FA in BME \& HC with its applicability are demonstrated in Sect. "Variants of FA used in BM and HC". Section "Applications of FA" describes the applications of FA in BM field as well as $\mathrm{HC}$ along with a discussion in design issues. Section "Critical Analysis" discusses the critical aspects of FA in BME and $\mathrm{HC}$ in all sorts. Research challenges along with some future directions have been discussed in Sect. "Research Challenges and Discussions". The conclusion of this paper along with some possible future issues is discussed in Sect. "Conclusion and Future Trend".

\section{Firefly Algorithm}

FA is originally proposed by Xin-She Yang [26, 27], based on the flashing behavior and models of Fireflies. It is renowned that the flash intensity varies with distance from the origin. Fireflies are usual example of the living being which utilizes bioluminescence for sexual selection.
Flashing, stable glows and chemical indication procedure are the different ways to communicate or attach with comparison in wooing (courtship). Many physical rules have been carried out by these flashing lights. In this way, mating process of firefly takes place.

The three idealized rules followed by FA are [28]:

1. Fireflies are attracted towards others regardless of their gender as they are unisex in nature.

2. The attractiveness of FA is relative with brightness of FA and they both reduce as their distance increases. So less attractive FA will move towards the more attractive FA. If there does not exist any brighter one as compared to a particular firefly, then the movement of the firefly will be random.

3. The brightness depends on the objective function.

\section{Structure of FA}

On the basis of the principle of intensity light 'I', Yang [8] introduced FA for which the inception of light diminishes with increase in ' $\mathrm{r}^{2}$ ', (square of the distance). As there is a speedy increase in origin of the light, the light shifts towards the unclear condition based on the light's observance (retention). To optimize, the present criterion is correlated with objective function. The disparity of light intensity as well as the attractiveness of FA became special attraction among the researchers and other experts. On the other hand, after FA's initiation, several improvements as well as transitions are made and FA is categorized to its nature and performance and is organized in the following way:

- Interpretation of fireflies.

- Computation of fitness function.

- Population procedure.

- Creation of novel best solution.

- Movable fireflies.

For decoding the related solution of a problem, FA has been extensively used especially for the problems of optimization. But, in most of the cases, the desired solutions cannot be resolved for a particular problem which is one of the features of no-free lunch theorem [29].

For the categorization of solutions, the value of ' $I$ ' in FA is united to coefficient of the fitness function [8] i.e.

$I(s) \propto F(s)$

where $I$ intensity, s solution, $F$ fitness function.

But, at the same time, the light intensity reconstructs from Eq. (1):

$I(r)=I_{0} e^{-a r^{2}}$ 
The deviation at $r=0$ in $I / r^{2}$ is refrained with the combination of inverse square law and Gaussian form's absorption.

The attractiveness of firefly is proportional to $I(r)$ i.e.,

$V \propto I(r)$

where $V$ attractiveness of the firefly, $I(r)$ intensity of light.

For determining $V$, the Eq. (2) can obstinate as Eq. (3)

$V=V_{0} e^{-a r^{2}}$

The light intensity as well as the brightness is mutually dependent with each other. As per the statistical FA, the distance of two fireflies' ' $k$ ' and ' $k$ ' in provisions of Euclidean distance is eq. (4)

$r_{i j}=\left\|k_{i}-k_{j}\right\|=\sqrt{\sum_{d=1}^{d=n}\left(k_{i d}-s_{j d}\right)^{2}}$

The movement of ith firefly is fascinated in the direction of other attractive firefly ' $j$ '. According to this criterion, Eq. (5) can be obtained.

$k_{i}=k_{i}+V_{0} e^{-a r_{i j}^{2}}\left(k_{j}-k_{i}\right)+j n_{i}$

where $n_{i}$, arbitrary value sapped from the Gaussian distribution.

The basic operation of Firefly algorithm is described as follows.

\section{Systematic Literature Review Process}

FA is one of the efficient yet popular nature inspired metaheuristic algorithm. FA attains popularity in prominent fields such as classification [65]. Likewise, FA has also been used in biomedical as well as health care areas. However, there is no such an article published that reviews the significance of FA in biomedical and health care fields. Therefore, taking these factors into consideration, in this article, an in-depth analysis has been made on the applications, advancements as well as challenges of FA in various applications. For this, we have used a special paper search strategy for paper collection. Keyword ("Firefly Algorithm in biomedical and health care") has been searched on benchmark databases such as Google Scholar, IEEE Xplore, Elsevier, Springer, Wiley, Inderscience, Taylor \& Francis. The paper search strategy has been depicted in Fig. 1. Based on the keyword search, we found 111 papers are related to FA in $\mathrm{BM} \& \mathrm{HC}$.

\section{Paper Inclusion Process}

To obtain exact papers related to study, we have performed keyword search on online databases and purely on the basis of keyword search, we found many papers. For accurately collecting records, we have applied paper inclusion process for filtering papers related to FA in BM \& HC. The paper inclusion procedure applied in this study is as follows:

\section{Basic Steps of Firefly Algorithm}

1. Initialize the population by randomly generated the Fireflies.

2. Initialize the FA algorithm parameters.

3. Calculate the fitness of all the Fireflies in the population by using defined objective function.

4. Find the attractiveness and relative distance between each pair of fireflies.

5. Update Fireflies positions.

6. Generate of new Fireflies and update the light intensities.

7. Rank the fireflies based in their fitness.

8. Select the better Fireflies for the next population as compared to previous Fireflies from old population.

9. Check the termination criteria. If it is true, then go to Step.10. Else, go to Step.3.

10. Return the best Firefly from the final population.

11. Exit 


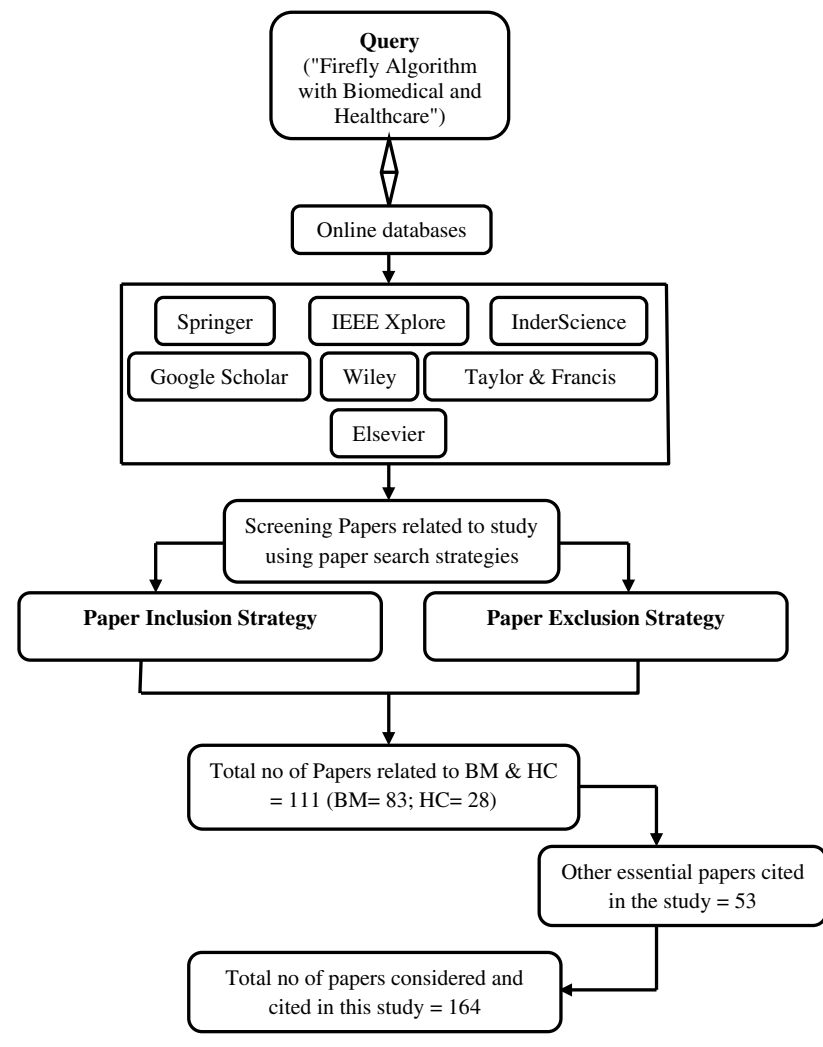

Fig. 1 Systematic literature review procedure considered for this study

1. Collecting Papers related to FA in BM \& HC.

2. Considering Survey Papers related to BM \& HC.

3. Considering Review Papers related to BM \& HC.

\section{Paper Exclusion Process}

During the process of paper collection, we found many papers related to FA in other applications rather than BM \& HC. Therefore, to screen such kind of papers which are not useful and not related for this study, we have applied exclusion procedure. The paper exclusion procedures applied in this study for filtering papers not related to FA in BM \& HC are considered as follows:

1. Removing Papers not related to study.

2. Screening Papers not relevant to FA.

3. Avoiding Papers or abstracts not accessible to read and download.

The total number of 164 papers are considered and cited for the final study. In those 164 papers, 111 papers are related to $\mathrm{BM} \& \mathrm{HC}$ and rest of 53 papers are necessary papers to consider but not related to MB \& HC. Out of those 111 papers, papers found that are related to biomedical field are 83 and papers related to health care field are 28 . The data taken from all the paper considered in this study are: dataset used, type of problem FA applied, variant of FA used, performance metrics used and usage levels of statistical FA $\&$ its variants etc.

\section{Variants of FA Used in BM and HC}

In last decades, the standard FA is found to be very successful and efficient in diversified domain. Furthermore, the researchers from optimization community have done continuous effort to improve the performance and convergence of the FA. With rapidly increasing literatures, more variations of FA are coming up day by day. Inspired from these facts, this study is dedicated for advances, issues and challenges of FA in biomedical and health care application. There are some improved versions of FA, developed with better performance for solving discrete problems which has already been used to solve travelling-salesman problems and other application domains [30]. Some of the developments of FA as per the criteria of controlling parameters are depicted in Table 1.

Also, extension of FA to multi objective optimization is examined in [14]. Some studies demonstrate that chaos has improved the performance of FA [48], while other studies have hybridized FA with some competitive algorithms to improve their performance [49-51]. Apart from these application domains, several variants of FA have been already applied to overcome some conflicts as well as for improving the performance of the current state-of-art of domain problems such as BM \& HC. The variations used for these types of areas have been explored in Fig 2. Also, modifications on the variants as well as the preliminary concept of specific variant have been demonstrated in Table 2. This section summarizes some of the frequently used variants of FA in BM \& HC fields.

\section{Hybridized Firefly Algorithm (HFA)}

FA has become a problem solver of global optimal solutions, but still it imposes some conflicts according to no-free lunch theorem [29]. To overcome these conflicts, hybridized FA's have been developed by combining FA with another algorithm which tends to create a new variation of FA. Now-adays, there has been a vital increase on the usage levels of hybridized versions of FA in the field of BM \& HC. Some of the previous literatures using HFA'S related to this field have been explained in this section. A novel methodology for determining parameter tuning in PSVM (Penalized Support Vector Machine) with the combination of FA with statistical PSO has been proposed by Al-Thanoon et al. [49] in the year 2019. Experimentations on several chemical datasets 
Table 1 Some important improvements in FA

\begin{tabular}{|c|c|}
\hline Controlling parameters & Improvement/modification \\
\hline Random movement parameter & $\begin{array}{l}\text { The Random Movement Parameter is improved [31] by including additional step for updating, which pro- } \\
\text { vides similarity and perform like PSO. This Modified FA has been successfully used in infinite impulse } \\
\text { response identification. This modification makes use of two parameters } \alpha_{0} \text { and } \alpha_{\infty} \text { in the place of } \alpha \text { (step size). } \\
\text { Another study [32] reported an improved FA with adaptive randomized movement parameter }(\alpha) \text { in which } \alpha \\
\text { are updated with iteration and maximum iteration. Further, similar improvement is made by Yu et al. [33] } \\
\text { incorporating randomized parameter using number of iteration. Yu et al. [34] has made an attempt to improve } \\
\text { random movement parameter by using solution's historic performance i.e. best position and global best solu- } \\
\text { tion till current iteration }\end{array}$ \\
\hline Attraction parameter & $\begin{array}{l}\text { Lin et al. [35] has improved the Attraction Parameter }(\beta) \text { by introducing virtual distance }(r) \text { to handle the } \\
\text { extreme change in attraction. Another study reported [36] the improvement of attraction parameter by using } \\
\text { light intensity function. They suggested that the selection of a direction from 'm' number of random direction } \\
\text { could be efficient and could handle the issue of degradation of brightness. However, this may lead to increas- } \\
\text { ing complexity of the algorithm. Gandomi et al. [37] and Khalil et al. [38] have modified } \beta \text { and } \gamma \text { by using } \\
\text { chaos function and chaotic mapping respectively }\end{array}$ \\
\hline $\begin{array}{l}\text { Random movement along with } \\
\text { attraction parameter }\end{array}$ & $\begin{array}{l}\text { A modified FA suggested by Yan et al. [39] to deal with higher problem dimension and feasible region, by } \\
\text { modifying the generalised brightness }(\beta) \text { by changing virtual distance }(r) \text { and step size }(\alpha) \text {. To enhance } \\
\text { exploration, Sulaiman et al. [40] has modified FA by using minimum variation distance in the place of Car- } \\
\text { tesian distance and mutation on } \alpha \text {. Another study [41] reported a novel way the handle premature conver- } \\
\text { gence by modifying } \alpha, \beta \text { and } \gamma \text { formulated light intensity difference. It is observed from this improvement that } \\
\text { brighter firefly has small attraction, and firefly with lesser brightness has randomness in step length. Othman } \\
\text { et al. [42] has modified FA by changing } \alpha \text { and } \gamma \text { in each iteration and by setting } \beta=1 \text {. Subramanian et al. [43] } \\
\text { has reported a modification on FA by adjusting } \alpha \text { by using } \alpha_{\min ,}, \alpha_{\min } \text {, iteration, and maximum generation. } \\
\text { Liu et al. [44] have modified the parameters } \alpha \text { and } \gamma \text { of FA by using }\left\{\alpha_{a}, \alpha_{e} \text {, iteration, and maximum genera- }\right. \\
\text { tion }\} \text { and }\left\{\gamma_{a}, \gamma_{e} \text {, iteration, and maximum generation }\right\} \text { respectively. In this study, it is reported that } \alpha \text { increases } \\
\text { and } \gamma \text { decreases with increase in iterations. Similar study [45] has been made where the parameter } \gamma \text { has been } \\
\text { adjusted by using } \gamma_{\min }, \gamma_{\max } \text {, iteration, and maximum generation, and then the } \gamma \text { is used to adjust } \alpha \text {. Fister } \\
\text { et al. [46] has modified } \alpha, \beta \text { and } \gamma \text { to make them self adaptive by using gross distribution and neighborhood } \\
\text { operator. Another study [47] suggested evaluating parameter } \gamma \text { by using Gaussian or normal distribution, } \\
\text { iteration and maximum generation. Also, this study suggested to modify } \alpha \text { by using } \alpha_{\text {initial }}, \alpha_{\text {final }}, \text { iteration, and } \\
\text { maximum generation }\end{array}$ \\
\hline
\end{tabular}

for evaluating the performance of the author's proposed methodology proved that the method have the capability for balancing suitable intensification and diversification and it is found to be efficient when compared to statistical PSO, FA as well as cross-validation (CV). It was also observed that the experimentations showed an enhanced accuracy rate in terms of classification rather than compared ones. Wang et al. [57] has proposed a collective mutation function into an enhance HFA, to efficiently consider the local as well as global search capabilities for resolving the optimization issues. As chaotic search (CS) reveals superior ergodicity, an evolution approach for arbitrary association of all fireflies according to CS was introduced, that improves the algorithm capability to negotiate the overall search space.

CDSS (Clinical Decision Support System) is one of the automated systems which became helpful in recent days to aid researchers as well as doctors of healthcare community. In the year 2018, for enhancing the CDSS query optimizer, Sharma et al. [50] has proposed a novel method by combining the CGA (Controlled Genetic Algorithm) with HFA. At first, the queries of big clinical data on the basis of TPC-DS was evaluated using RDFG-CDQO (a restricted diverge environment). Then, a performance comparison is measured with some other standard techniques: RS-CDQO etc. The authors made an analysis that the proposed method yields an enhanced performance other than the compared techniques. To detect the severe yet repeated disease named MIF (Myocardial Infarction), Kora [51] has proposed a modern method by hybridizing the HFA with PSO on the basis of ECG method in the year 2017. For the evaluation of the capability of the proposed method, the author considered sensitivity, specificity as well as classification accuracy as a performance factors. A comparison has been made among three classifiers such as RAW ECG, Filtered ECG and ECG beat for validating the performance and also it has been compared with some different standard techniques such as MRI (magnetic resonance imaging) etc. Higher classification accuracies have been found to be of $99.3 \%, 99.97 \%$ and $98.7 \%$ in terms of accuracy rate, sensitivity and specificity respectively. Some additional developments using HFA have been depicted in Table 3.

\section{Modified Firefly Algorithm (MFA)}

Since from its initiation, many researchers have attempted several techniques for enhancing the performance of 


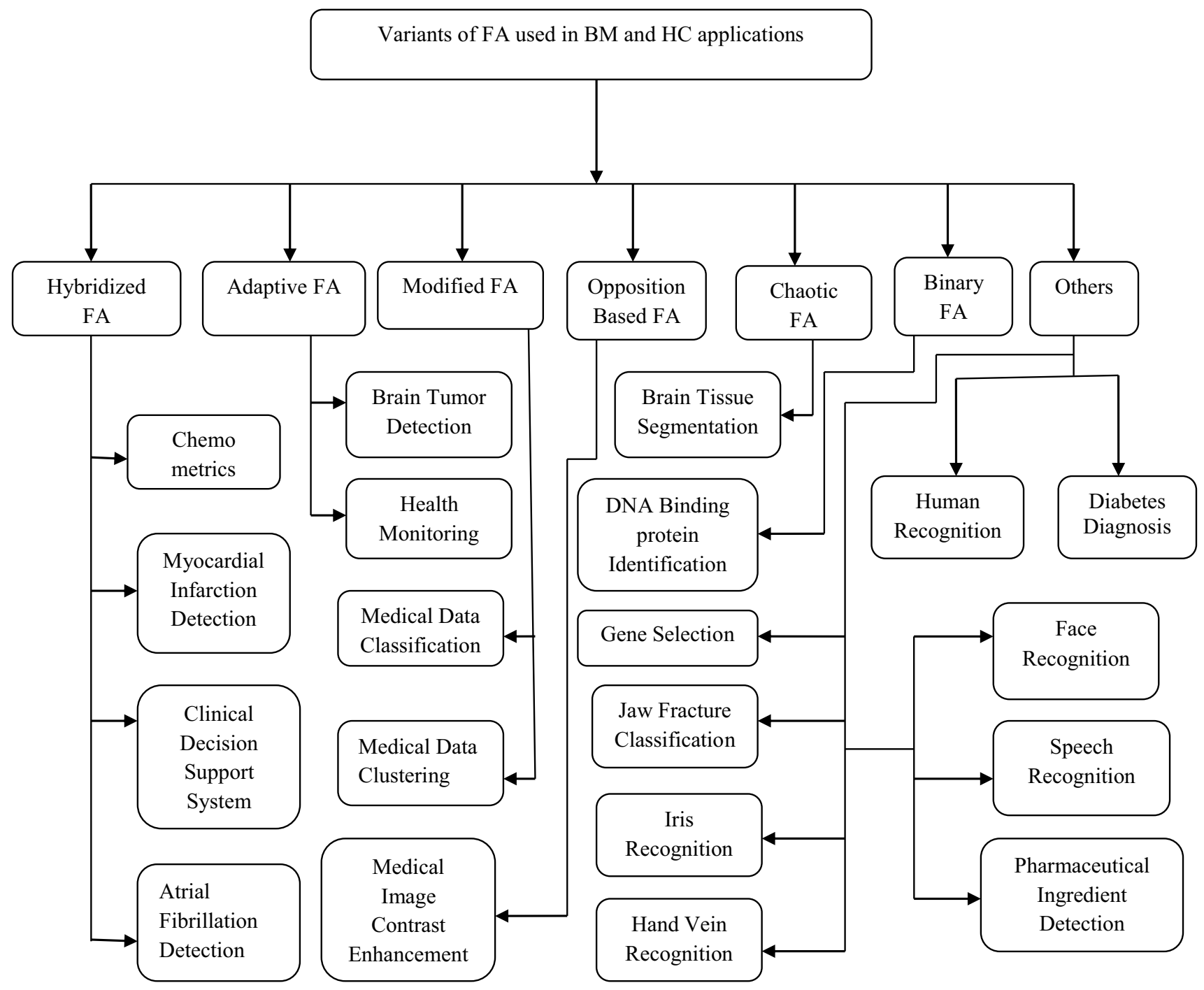

Fig. 2 Variations of FA utilized in Biomedical and Healthcare

Table 2 Different variants of FA

\begin{tabular}{|c|c|c|c|c|}
\hline S.no & Variant name & Concept behind the variant & Modification in parameters & References \\
\hline 1. & Hybridized FA & FA combined with IFCM & Optimal membership matrix and cluster center & {$[52]$} \\
\hline 2. & Chaotic FA & Embedding of Chaotic map in firefly algorithm & $\begin{array}{l}\text { Replacing the random variables with chaotic vari- } \\
\text { ables }\end{array}$ & [48] \\
\hline 3. & Binary FA & $\begin{array}{l}\text { Use of normalized Hamming distance for computing } \\
\text { attractiveness }\end{array}$ & $\begin{array}{l}\text { Dynamic mutation operator for increasing diversity } \\
\text { of fireflies }\end{array}$ & {$[53]$} \\
\hline 4. & Modified FA & $\begin{array}{l}\text { Inclusion of wrapper based feature selection with } \\
\text { binary firefly }\end{array}$ & $\begin{array}{l}\text { Feature subset evaluation by SVM } \\
\text { Use of normalized hamming distance for attractive- } \\
\text { ness } \\
\text { Dynamic mutation operator for diversity }\end{array}$ & {$[54]$} \\
\hline 5. & Adaptive FA & Use of mediator model in firefly algorithm & Use of mediator model to adjust the parameters & {$[55]$} \\
\hline 6. & Opposition & Opposition based learning & $\begin{array}{l}\text { Hybridization of opposition based learning with } \\
\text { firefly algorithm }\end{array}$ & {$[56]$} \\
\hline
\end{tabular}




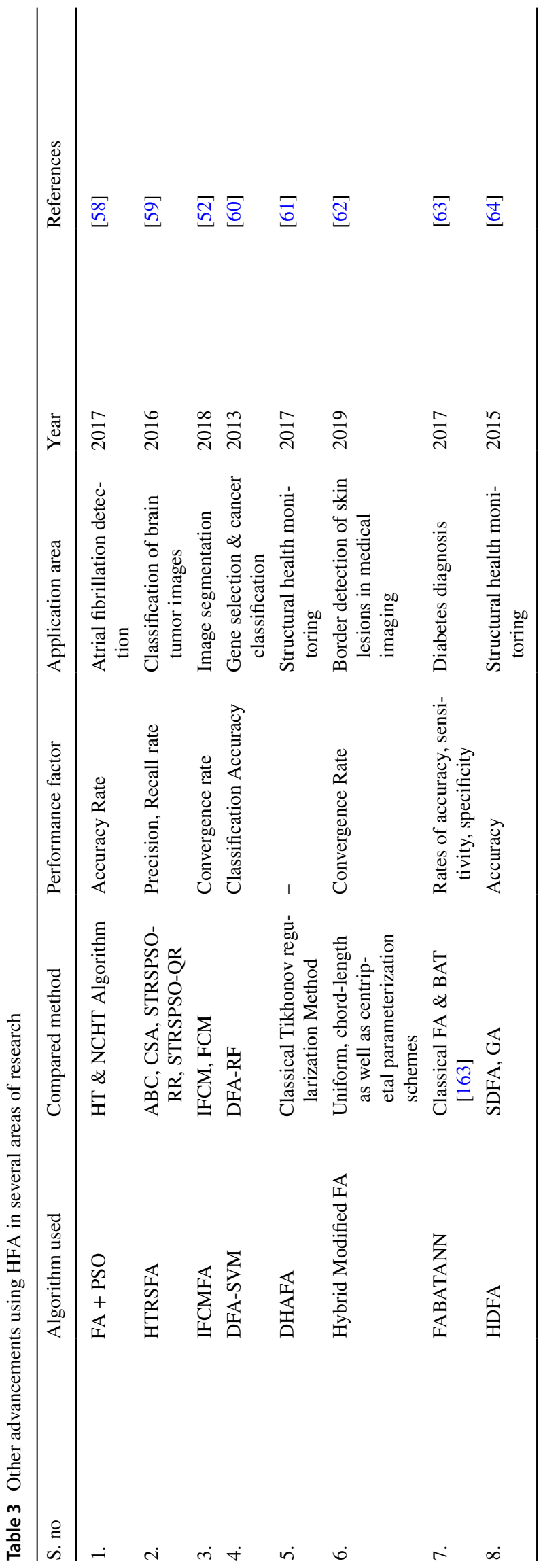

classical FA. Some major modifications have been proposed especially towards the change in parameter. According to the literatures [65], MFA has found to be one of the efficient versions of FA. MFA has been already applied to solve several problems of thoroughly used domains of research. According to our survey, we found that MFA has rare usage in the applications of BM \& HC. To reduce the complexity of classification for storing the huge amount of medical analysis data, Sahmadi et al. [54] has developed a novel way of approach with a MFA named BFA (Binary Firefly Algorithm) by combining it with SVMC (Support Vector Machine Classifier). For predicting the performance, several benchmark datasets has been considered such as breast cancer, diabetes etc. Population size, cross-fold validations etc are used as performance factors and the developed method was compared with other standard techniques such as GA [2], mimetic algorithm (MA) [66], harmony search algorithm (HSA) [67]. The authors concluded that their method yielded enhanced learning capability as well as stability for the estimation of the model. In 2016, Yuan et al. [68] has developed a MFA, which is a combination of FA and SAM (simulated annealing method) for the clustering of $\mathrm{CM}$ (Chinese Medicine) prescriptions. With the change in fitness, it is observed an enhanced capacity of global search strategy. Later, the projected method was compared with the original K-Means clustering algorithm and it is obtained that improved time for running the method has been found with better analysis of clustering, when compared to other considered method.

\section{Binary Firefly Algorithm (BFA)}

FA is positively affluent in many aspects. BFA is one variant of FA, has been utilized for solving some difficult yet tricky real world problems. Also, it has been used in BM \& HC domains. Zhang et al. [53] has introduced a novel method for the identification of DNA-binding proteins using BFA as well as MFF (Multi Features Fusion). On the basis of SVM with BFA, the method has been analyzed by the combination of parameters of optimization and features. Some popular yet standard algorithms such as GA, FA, BPSO etc have been taken into consideration for comparing the performance of present method. Later, the authors concluded that they found a very high rate of accuracy rather the compared techniques.

\section{Adaptive Firefly Algorithm (AFA)}

Major techniques are lacking in accurateness and some other limitations such as time \& computational complexity. To get out of all these drawbacks, a new variant called adaptive FA has been developed. Interestingly, AFA has been applied to BM \& HC field along with some other domains. For enhancing the detection as well as classification of brain tumor 
disease, Deepa and Emmanuel [55] have proposed a novel method named FFABPNN (Fused Feature Adaptive Firefly Back Propagation Neural Network). Grouping of feature extraction, feature fusion, feature selection, feature classification have been employed for extracting the enhanced rate of classification accuracy. The new variant, AFA was extensively used for classifying the tumor as well as non tumors cells in an effective manner. The images from BRATS dataset has been considered to evaluate the performance of the developed model. Later, on the basis of experimental results, the authors concluded that the method yielded better performance for classifying the MRI tumor.

\section{Chaotic FA (CFA)}

FA is a very effective swarm intelligence based algorithm. However, chasing the local optima results was one of the challenging aspects for FA. As a result, a new variation such as chaos based FA is proposed with the integration of chaos theory and FA. In the year 2018, for the segmentation analysis of tissues in human brain images of MRI, by using a variant of FA named CFA with the conventional FCM (Fuzzy C-means) has been developed by Ghosh et al. [48] and named the method as CFAFCM (Chaotic Firefly integrated Fuzzy C- means) algorithm. The concerned method has been tested over standard datasets of $20 \mathrm{MRI}$ images. After simulating the experimentations, a comparison is made with other recent developed techniques such as FCM, BCFCM etc. The authors concluded that the developed method has higher performance with the factor such as rate of convergence etc and also found a conflict with the usage of CMs.

\section{Applications of FA}

FA is one of the well-liked nature inspired metaheuristic optimization that has been extensively used from its initiation to the present days to elucidate several problems of different application domains. FA has been successfully applied to optimization, data clustering, classification etc and many variants of FA are used to solve different complex problems. Further, it has been emerged as one of the successful metaheuristic for BME \& HC. This section demonstrates the major applications of FA that has been used frequently in $\mathrm{BM} \& \mathrm{HC}$.

\section{FA in Biomedical Engineering}

Sánchez et al. [69] has introduced a novel approach for modular neural network (MNN) design using FA and granular computing which is tested with human recognition based on standard face and ear databases to confirm the efficiency and the advantages. Authors have compared the methods with their attained products for ear and face recognition against a hierarchical GA to know the improved results for human recognition. Reducing the recognition error is the main motive of author's projected method. A novel approach for the computer diagnosis of breast cancer has been developed by Krawczyk and Filipczuk [70] which is completely dependent on the nuclei exposure using FA, one-class disintegration approach and marker controlled break point segmentation. Authors have tested their solution on a cytological image sets attained through regular hospital work. They have used decomposition of hybrid one class categorization as the pattern detection module which guides to scientific and statistically noteworthy development over widely used techniques. Author's projected framework subordinates an excellent rate of cancer detection and has been used as an important decision support system for each day clinical practice. Rahebi and Hardalaç [71] have presented an innovative computerized technique of optic disc recognition in human retinal images by utilizing the FA. The author's major concern was developing FA to recognize the optic disc in retinal images. By using their technique, some elements have been arbitrarily applied to retina images at original state and every pixel was measured as firefly lightening. Formulation of insect's desirability and alter in light intensities of image pixels are the two key subjects that are concerned in their anticipated technique. Intensity of light is proportional to fitness of insects where attraction is a comparative parameter and was assessed by the other insects. Sawhney et al. [72] has developed a firefly-based wrapper feature collection technique using random forest classifiers for cancer diagnosis. Authors have customized the active fitness functions with the addition of a penalty factor to endorse quality reduction. They have also made a relative study of existing SI based feature selection techniques in terms of feature reduction and accurateness. Their novel penalty function appeared to be highly effective in decreasing the characteristics which saves the computational transparency and conflict the annoyance of dimensionality on classification accuracy. Many other applications that have been utilized with FA and its variants have been depicted in Table 4 .

\section{FA in Biomedical Signal Processing}

Biomedical signal processing (BSP) is the surveillances of the physiological behavior of individuals, varies from sequences of protein as well as gene to neural rhythms, organ, and tissue images. Biomedical signal processing intends to extort major data from biomedical signals. Biologists can determine a novel physician and biology to handle discrete illnesses with the assistance of BSP. SI based algorithms such as FA, EA, PSO, and ABC, etc could signify an appropriate method for the alignment of biomedical signal processing. 
Table 4 Applications of FA in BM field

\begin{tabular}{|c|c|c|c|c|c|c|}
\hline S. no & Type of FA used & Application type & Dataset used & Compared method & Result & References \\
\hline 1 & Chaotic FA & $\begin{array}{l}\text { Brain tissue segmenta- } \\
\text { tion }\end{array}$ & IBSR Dataset & $\begin{array}{l}\text { SGFC (Stochastic gra- } \\
\text { dient descent based } \\
\text { fuzzy clustering) }\end{array}$ & $\begin{array}{l}\text { Good segmentation } \\
\text { rate }\end{array}$ & {$[48]$} \\
\hline 2 & Hybrid FA & Chemometrics & QSAR dataset & PSO, Statistical FA & $\begin{array}{l}\text { Superior performance } \\
\text { in terms of accuracy }\end{array}$ & [49] \\
\hline 3 & Levy- Flight FA & $\begin{array}{l}\text { Radio therapy treat- } \\
\text { ment }\end{array}$ & CT Image dataset & Statistical FA & $\begin{array}{l}\text { Better accurate results } \\
\text { than other methods }\end{array}$ & [73] \\
\hline 4 & Moth FA & $\begin{array}{l}\text { Facial emotion recog- } \\
\text { nition }\end{array}$ & JAFFE, MMI etc & GA, PSO etc & $\begin{array}{l}\text { Moth FA yield better } \\
\text { performance among } \\
\text { compared ones }\end{array}$ & [74] \\
\hline 5 & Hybrid FA & $\begin{array}{l}\text { Atrial fibrillation } \\
\text { detection }\end{array}$ & $\begin{array}{l}\text { Atrial Fibrillation } \\
\text { Dataset }\end{array}$ & $\begin{array}{l}\text { HT (Hadamard } \\
\text { Transform), NCHT } \\
\text { (Natural Ordered } \\
\text { Complex Hadamard } \\
\text { Transform) }\end{array}$ & $99.3 \%$ detection rate & {$[58]$} \\
\hline 6 & Hybrid FA & Image segmentation & $\begin{array}{l}\text { MRI scan, Rice, Lena } \\
\text { and satellite image }\end{array}$ & $\begin{array}{l}\text { IFCM (Improved } \\
\text { Fuzzy C-Means), } \\
\text { FCM (Fuzzy } \\
\text { C-Means) }\end{array}$ & $\begin{array}{l}\text { IFCMFA yields higher } \\
\text { performance than } \\
\text { IFCA }\end{array}$ & {$[52]$} \\
\hline 7 & Modified FA & $\begin{array}{l}\text { Medical data classifi- } \\
\text { cation }\end{array}$ & $\begin{array}{l}\text { Heart, Diabetes Breast } \\
\text { Cancer datasets etc }\end{array}$ & SVM & $\begin{array}{l}\text { Better classification } \\
\text { accuracy }\end{array}$ & {$[54]$} \\
\hline 8 & Modified FA & Clustering & $\begin{array}{l}\text { Medical prescription } \\
\text { data }\end{array}$ & FS-K-Means & $\begin{array}{l}\text { FA-K-Means yields } \\
\text { better performance } \\
\text { than FS-K-Means }\end{array}$ & {$[68]$} \\
\hline 9 & Classical FA & $\begin{array}{l}\text { Pseudo relevance } \\
\text { feedback }\end{array}$ & MEDLINE dataset, & $\begin{array}{l}\text { RSJ and Rocchio } \\
\text { methods }\end{array}$ & $\begin{array}{l}\text { Superior Performance } \\
\text { with FA }\end{array}$ & {$[75]$} \\
\hline 10 & FA & $\begin{array}{l}\text { Brain region segmen- } \\
\text { tation }\end{array}$ & Brain MRI dataset & - & $\begin{array}{l}\text { Enhanced Segmenta- } \\
\text { tion Accuracy }\end{array}$ & [76] \\
\hline 11 & FA & Lungs segmentation & $\begin{array}{l}\text { JRST and Montgomery } \\
\text { County }\end{array}$ & SIFT-Flow, SCAN & $\begin{array}{l}95.1 \% \text { segmentation } \\
\text { accuracy }\end{array}$ & {$[77]$} \\
\hline 12 & Binary FA & $\begin{array}{l}\text { DNA protein identifi- } \\
\text { cation }\end{array}$ & Protein Data Bank & Statistical FA & $\begin{array}{l}\text { Binary FA yields } \\
\text { better performance } \\
\text { than Statistical FA in } \\
\text { identifying protein }\end{array}$ & {$[53]$} \\
\hline 13 & FA & $\begin{array}{l}\text { CT/MR Image Seg- } \\
\text { mentation }\end{array}$ & Abdomen dataset & $\begin{array}{l}\text { ABC, Cuckoo Search } \\
\text { Algorithms }\end{array}$ & $\begin{array}{l}\text { Good segmentation } \\
\text { rate }\end{array}$ & [78] \\
\hline 14 & FA & Feature Selection & $\begin{array}{l}\text { BCI competition } \\
\text { dataset }\end{array}$ & $\begin{array}{l}\text { Sequential Forward } \\
\text { Search, GA }\end{array}$ & Superior performance & [79] \\
\hline 15 & DHAFA & Health Monitoring & Not defined & Adaptive FA & $\begin{array}{l}\text { DHAFA acts as an effi- } \\
\text { cient tool for health } \\
\text { monitoring }\end{array}$ & {$[61]$} \\
\hline 16 & FA & $\begin{array}{r}\text { Water Marking of } \\
\text { Medical images }\end{array}$ & RONI data & $\begin{array}{l}\text { DWT-DCT based } \\
\text { methods }\end{array}$ & Standard performance & {$[80]$} \\
\hline 17 & HDFA & Health monitoring & Not defined & SGA, SDFA & $\begin{array}{l}\text { HDFA outperforms the } \\
\text { SDFA and SGA }\end{array}$ & {$[64]$} \\
\hline 18 & Levy Flight FA & $\begin{array}{l}\text { Segmentation of Medi- } \\
\text { cal Images }\end{array}$ & CECT image dataset & $\begin{array}{l}\text { Traditional ACM } \\
\text { (Active Contour } \\
\text { Models) }\end{array}$ & $\begin{array}{l}\text { ACM-LFA outper- } \\
\text { forms Traditional } \\
\text { ACM }\end{array}$ & [81] \\
\hline 19 & FA & $\begin{array}{l}\text { Electrophoresis of } \\
\text { images }\end{array}$ & GE image dataset & - & $\begin{array}{l}\text { Good DNA separa- } \\
\text { tion rate has been } \\
\text { obtained }\end{array}$ & {$[82]$} \\
\hline 20 & Back Propagation FA & $\begin{array}{l}\text { Epilepsy detection of } \\
\text { EEG }\end{array}$ & EGG signal data & - & $\begin{array}{c}93.3 \% \text { detection rate } \\
\text { has been obtained }\end{array}$ & {$[83]$} \\
\hline 21 & FA & $\begin{array}{l}\text { Epilepsy classification } \\
\text { of EEG }\end{array}$ & $\begin{array}{c}\text { EEG recordings of } 20 \\
\text { patients (Sri Ram- } \\
\text { akrishna Hospital, } \\
\text { Coimbatore, India) }\end{array}$ & - & $96.07 \%$ accuracy & [84] \\
\hline
\end{tabular}


Table 4 (continued)

\begin{tabular}{|c|c|c|c|c|c|c|}
\hline S. no & Type of FA used & Application type & Dataset used & Compared method & Result & References \\
\hline 22 & FA & $\begin{array}{l}\text { Registration of medical } \\
\text { image }\end{array}$ & $\begin{array}{l}\text { Human Head CT } \\
\text { Image data }\end{array}$ & Powell optimization & Superior performance & [85] \\
\hline 23 & FA & Face recognition & Yale face database & GA, PSO & $99.16 \%$ accuracy & [86] \\
\hline 24 & FA & Emotion recognition & EEG data & $\mathrm{RBF}$ & $\begin{array}{l}\text { ISO-FLANN with FA } \\
\text { outperformed over } \\
\text { compared method }\end{array}$ & [87] \\
\hline 25 & FA & Facial recognition & $\begin{array}{l}\text { CK+ (Cohn-Kanade), } \\
\text { JAFFE databases }\end{array}$ & GA, PSO & $98.45 \%$ accuracy & [88] \\
\hline 26 & FA & Speech recognition & $\begin{array}{l}\text { Synthetic data (Own } \\
\text { data) }\end{array}$ & PSO & FA outperforms PSO & [89] \\
\hline 27 & Compact FA & $\begin{array}{c}\text { Pharmaceutical ingre- } \\
\text { dient determination }\end{array}$ & Escitalopram data & GA & CFA outperforms GA & {$[90]$} \\
\hline 28 & HMFA & $\begin{array}{l}\text { Border detection of } \\
\text { medical images }\end{array}$ & Skin Lesion Images & - & Superior performance & {$[62]$} \\
\hline 29 & FAFCM & $\begin{array}{l}\text { MRI brain segmenta- } \\
\text { tion }\end{array}$ & MRI Image dataset & Traditional FCM & $\begin{array}{l}\text { Better segmentation } \\
\text { rate }\end{array}$ & [91] \\
\hline 30 & Hybrid FA & $\begin{array}{l}\text { Myocardial infarction } \\
\text { detection }\end{array}$ & Diabetes dataset & $\begin{array}{l}\text { Traditional FA, Bat } \\
\text { algorithms }\end{array}$ & $\begin{array}{l}\text { Hybrid FA outper- } \\
\text { forms compared } \\
\text { methods }\end{array}$ & {$[63]$} \\
\hline 31 & FA & Hand vein recognition & NCUT dataset & $\begin{array}{l}\text { Euclidean, Hausdorff } \\
\text { distance etc }\end{array}$ & $\begin{array}{l}\text { FA outperforms com- } \\
\text { pared methods }\end{array}$ & [92] \\
\hline 32 & $\begin{array}{l}\text { FA+ SEFNN (Struc- } \\
\text { ture Equivalent Fuzzy } \\
\text { Neural Network) }\end{array}$ & Speech recognition & Speech data & $\mathrm{PSO}+\mathrm{FNN}$ & Higher recognition rate & {$[56]$} \\
\hline 33 & FAFCM & $\begin{array}{l}\text { MRI Brain Image } \\
\text { Segmentation }\end{array}$ & MRI Image data & FCM & $\begin{array}{l}\text { FACFM outperforms } \\
\text { FCM }\end{array}$ & [93] \\
\hline 34 & FA & $\begin{array}{l}\text { Data Classification \& } \\
\text { Disease Diagnosis }\end{array}$ & IRIS, Wine, Heart etc & $\mathrm{PSO}$ & $\begin{array}{l}\text { Better classification } \\
\text { rate }\end{array}$ & [94] \\
\hline 35 & FA & $\begin{array}{l}\text { MRI Brain Image } \\
\text { Segmentation }\end{array}$ & BraTS 2D MRI dataset & - & $\begin{array}{l}\text { Better Segmentation } \\
\text { rate }\end{array}$ & [95] \\
\hline 36 & Opposition based FA & $\begin{array}{l}\text { Medical Image Con- } \\
\text { trast enhancement }\end{array}$ & Medical Images & $\begin{array}{l}\text { FFA, AMFF (Adaptive } \\
\text { Moment Fireflies) }\end{array}$ & $\begin{array}{l}\text { Better Contrast } \\
\text { enhancement rate }\end{array}$ & [96] \\
\hline
\end{tabular}

Biomedical ontologies have achieved meticulous significance in the area of science because of its major part in representing data in this domain. Being motivated by the achievement of FA in several areas such as big data optimization, task scheduling, and brain image segmentation, etc, Xue [97] has introduced a compact firefly algorithm (CFA) that uses the clear depiction of the population with a possibility distribution, and bio-compact movement functions to progress the matching competence and guarantee the quality of alignments. To test the performance of CFA, the author has developed the anatomy track, disease, biodiversity, disease, ecology, and phenotype track from the ontology alignment evaluation initiative (OAEI). A biomedical model resemblance metric was proposed by the authors to assess the similarity rate of two biomedical perceptions. Based on the experimentation, the authors have concluded that CFA can enhance the FA based matches memory usage with $68.92 \%$ and runtime with $38.97 \%$ as average. The categorization of uterine magnetomyography (MMG) signals was endeavored to describe the reduction of uterine that escorted to true labor. Kumar and Rajesh [98] have attempted to optimize the Physionet features that are extorted with six-level discrete wavelet transform (DWT) techniques for several wavelets. The authors have analyzed the Physionet mmgdb database by using the DWT. The chosen features then supplied to four various classifiers for the labor evaluation. The individual performance of the classifier was considered for several mother wavelets. The authors have compared the result of their experimentation with PSO and GA. The distinct wavelet features optimized with FA, and extorted with the coif5 wavelet has outperformed better than the remaining algorithms. Based on the experimentation, the authors have concluded that the SVM classifier has produced better accuracy, precision, and false-positive rate with $97.2105 \%$, $97.3108 \%$, and $5.3784 \%$ respectively. The outcomes that are attained from their study aids clinicians in considering the process of parturition, and labor assessment. The advancement suggested by the authors has the prospective to attain this in the field of labor evaluation for both terms as well as preterm deliveries. Cardiotocography scrutinizing is a fetal 
state measurement technique. The heart rate recording as well as a uterine contraction in the period of pregnancy can be controlled by CTG. The outcomes of CTG permit the detection of three essential several fetal states such as normal, suspected, and pathological and the medicine to identify and evaluate conditions that may direct to fetal death. The recorded information with CTG can assist gynecologists to assess the security of the fetus due to the complexity of access the fetus unswervingly. Ali et al. [99] has proposed an ensemble learning relay on an assortment of features and a categorization ensemble technique to enhance the accuracy. The authors have used FA for choosing the best division of attributes from CTG dataset. They have utilized the $\mathrm{KNN}$ to categorize normal, suspicious as well as pathological models that rely on the extorted features from cardiotocograms. Based on the results, the authors have concluded that the KNN classifier can effectively distinguish these classes with a total $93.3 \%$ accuracy of classification. Cancer is an identified cause of fatality worldwide. Certain analysis of cancer insists on the categorization of various kinds of tumor. Explorations into microarray DNA terminology appear to be a winning platform for altering genetic diseases. Though the benchmark ML advances have been effective in the understanding of major genes as well as categorization of new kinds of cases, some drawbacks in the logical and medical applications has confronted that includes DNA microarray data examination restriction with an absurd quantity of features and a comparatively small volume of an example respectively. To attain information about practical and effective DNA microarray datasets, there was a requirement of enlarging the rank of prediction as well as interpretability advance during retaining an immense rate of precision. Jabar and Saadya [100] have presented a new kind of cancer categorization relay on gene expressions. The authors have used both mutual information and FA. They have utilized parameters for the selection of features before usage of FA for feature reduction. SVM was utilized to categorize cancer into various kinds. The authors were evaluated the performance of the proposed method with the colon cancer data and compared the attained outcomes with other new advances. From the experimentation, the authors have concluded that the projected technique can increase the accurateness of cancer categorization and reduce the quantity of chosen genes contacted by some other techniques. Electrocardiographic (ECG) signal is important to identify cardiac arrhythmia amid a variety of biological signals. The correct investigation of loud ECG signals was an extremely rousing challenge. Proceeding to automatic study, the sounds present in the ECG signal required to be eradicated for exact analysis. Most researchers have reported various techniques for denoising the signals of ECG in current years. So, Sundararaj [101] has proposed an optimized threshold instrument for wavelet relied on health signal noise decline. The process completely depended on a variable step size FA (VSSFA) in a doubletree difficult wavelet method. The authors have assessed the projected advance on various usual as well as unusual ECG signals of BIH or MIT arrhythmia database, by accumulating white Gaussian noises synthetically with the difference of 5 and $10 \mathrm{~dB}$. The results were proved that the projected scheme was well concerted in several noise levels, and attain improved visual class contrast with additional techniques. From the experiments, the authors have concluded that the projected technique can decrease sound from noisy ECG signals more precisely in a constant fashion. Mining of fetal electrocardiogram (FECG) from the abdominal section of the mother's fur is a confront task because of the high overlie of fetal as well as maternal signals. To overcome the issue, Akhavan-Amjadi [102] has proposed the usage of extreme learning models (ELM) to teach the signals of FECG extorted by the least mean square (LMS) advance from the key thoracic as well as abdominal signals. The authors have used the skilled ELM techniques to form the FECG signal for checking the models. They have examined the FA to parameter tuning of ELM, and enhance the performance respectively. The projected method has implanted the evolutionary system into the FA that has advantages from mutation possibilities and adaptive crossover because of elevated difficulty as well as several FA parameters. The authors have concluded that the outcomes proved the capacity of the proposed technique when the abdominal signal comprises greater overlapping amid the fetal and maternal signals. Image segmentation is a basic step for image dispensation of health images. Border renovation is one of the major charges that comprises building a border curve untying the tissue as well as an organ of concern from the image background. Such a procedure can be invented as a problem of optimization that calculates the border curve with the process of information fitting from gathered data that implicit to recline on the border of the object in the analysis. Yet, normal mathematical optimization methods won't offer acceptable resolutions. A few latest articles have been utilized in evolutionary methods to defeat the risen problems. Gálvez et al. [103] has presented all these raised issues with the rational Bézier curves by using FA. Based on the experimentation, the author has concluded that the proposed technique has outperformed better results. El-Tokhy [104] has introduced an X-ray as well as decisive neutron radiography images firmness with $\mathrm{FA}$ and $\mathrm{ABC}$ algorithms. The authors have used these algorithms in combination with projected image encoding or decoding as well as disintegration or decompression algorithms. The encoding or decoding algorithms relays on hierarchical trees, binary images, and Huffman. Besides, disintegration or decompression algorithms utilize the Gaussian pyramidal transform, Hilbert transform, and real dual-tree transform. The major purpose behind the FF is the usage of Optimum 
compression ratio (OCR) with the preeminent radiography image eminence. The optimum rates of $\mathrm{ABC}$ for both X-ray and neutron images are $80.3610 \%$ and $80.3610 \%$ through hierarchical trees by the Hilbert transform. On the other hand, FA attained $97.0499 \%$ optimum value for the hierarchical trees by the Hilbert transform. Based on the experimental results, the author has concluded that the FA was renowned to achieve higher outcomes than the $\mathrm{ABC}$ algorithm for compacted radiography images. Moreover, some other applications of biomedical signal processing using FA are depicted in Table 5.

\section{FA in Medical Image Processing}

The method of performing a few functions on an image to attain an improved image or to extort some useful data can be considered as image processing (IP). IP is a kind of signal processing (SP), where an input can be an image and output will be either an image or associated features of the image respectively. Generally, an IP includes the importing of an image through image acquisition techniques, assessment as well as alteration of the image, and consequences in outcomes can be modified image on the analysis of the image. Analog and digital image processing are the two kinds of IPs, where Analogue image processing (AIP) can be utilized for photographs and printing of hard copies, and Digital image processing (DIP) methods assist in exploitation of the digital images with computers. FA is one of the appropriate algorithms for IP due to its faster convergence rate and lower computational time. FA in IP can improve the effectiveness of the procedural optimal searching methods. Various phases that all kind of information has to undergo while utilizing IP methods are registration, pre-processing, restoration, detection, segmentation and classification.

\section{FA in Medical Image Pre-processing}

Image pre-processing (IPP) is an enhancement of the image that contains reluctant alterations or improves a few image characteristics. An efficient preprocessing stage is necessary to attain higher detection rates. IPP plays a vital role in many fields, mainly in the health domain. Various techniques of IPP algorithms include image thresholding, page segmentation, skew alteration, noise deduction, and precise image augmentation. FA can be used in preprocessing of an image to attain better performance in denoising and reduction of noise in an image respectively.

Denoising an image has been playing a vital role in the area of Medical Imaging (MI). The deciding of informationbearing structures like surface as well as edges to attain proper visual eminence PSNR was the major challenging task. An innovative bio-inspired optimization by using MI was considered by Elhoseny and Shankar [110] and known

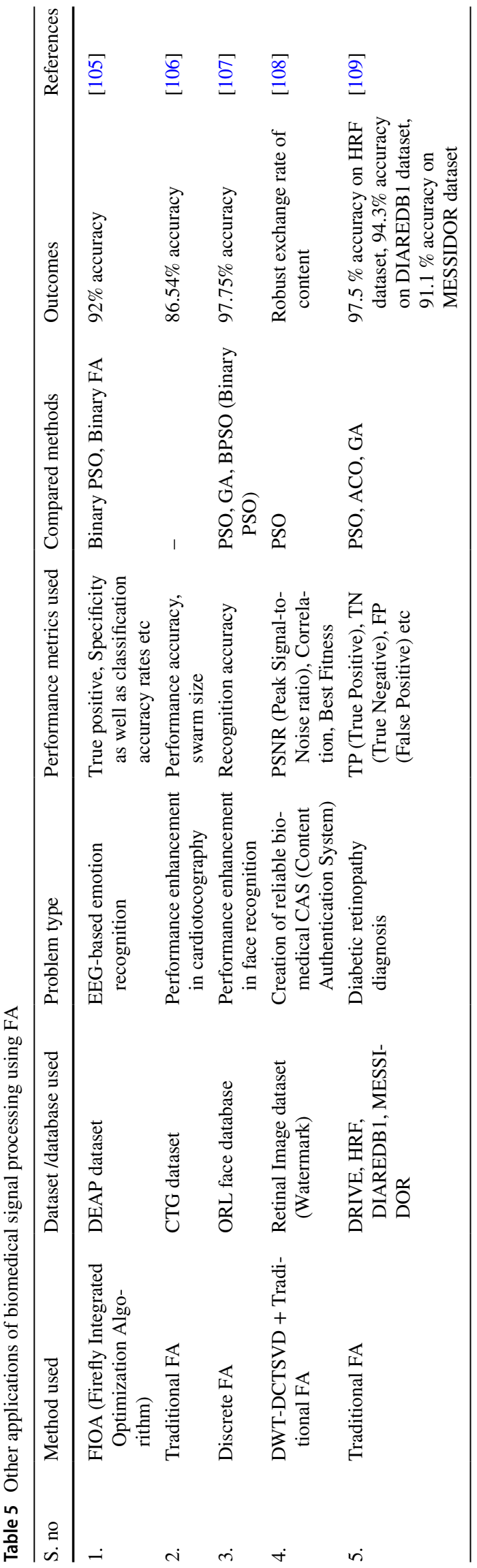


as Bilateral Filter (BF) respectively. The implementation of the denoising procedure influenced the result of choosing the optimal limitations such as spatial and Gaussian weights. These limitations were selected by using MFF as well as DF which are SI based methods. They have utilized the VRMSE as well as PSNR for the selection of parameters. Also, the CNN classifier was utilized to categorize the denoised image as usual or unusual with an accurate classification rate respectively. The authors have concluded that the projected technique has attained $47.52 \mathrm{~dB}$ of PSNR and 1.23 of error rate respectively. Image preprocessing supposes a vital role in various domains, particularly in medical domains where exact image details were needed for diagnosis, determination as well as research purposes, and many more. Some examples of Medical images such as MRI, X-ray, and CT have a few defects that they generate images along with noise. This produced noise appears due to transmission, poor enlightenment, and camera sensor cells respectively. So, Sam and FRED [111] have used a merge of filters to review the images through the noise. The considered Metaheuristics were used by the authors to train FFNN. So the projected methods reconfirm the denoised image respectively. The performance of noise filtering in MI was enhanced by utilizing NFN with the grouping of NF as well as post-processor (PP). Pugalenthi et al. [112] has introduced the FA-based hybrid NF filter to enhance the performance of noise reduction respectively. The projected noise reduction merges the benefits of fuzzy, neural, as well as FA. Besides, the authors have used an enhanced version of FA named penetrating based PSFA to illustrate the optimal shape of link function, and to lessening the local tripping issue. Based on the experimentation, the authors have shown the results that the projected method has proved its efficiency in decreasing the urge noise in MI against various impulse noise bulk ranks. Zhang et al. [113] introduced a new technique to extort end-members (EE) from hyper spectral images that relay on discrete FA (EE-DFA). To get better outcomes, the authors have utilized extort endmembers from the hyper spectral image and were considered as the problem of combinational optimization which can be resolved by DFA. They have considered various datasets for the series of experiments such as SNR and the AVIRIS Cuprite. It was found to be contrasted with the four various techniques such as SMACC, VCA, N-FINDR, and MVCNMF. Based on the experimentation, the authors have shown that the outcomes were illustrated to be outperformed better than the obtainable popular techniques. The anticipated EEDFA technique was strong with the sharp reduction of the SNR image.

\section{FA in Medical Image Restoration}

The process of obtaining a noisy as well as disintegrate image, and evaluating the exact original image is known as image restoration. Image restoration can be executed by spinning around the process that distorted the image. Makeup or undo the deficiency that humiliates an image is the major cause behind the image retrieval. The module is to decrease the Gaussian noise that was identified in MI and to attain improved picture quality respectively. Csam et al. [114] has introduced the computer-assisted model for identifying ominous and amiable liver tumors from CT images. The authors have projected the different hybrid methods for the emigration of the assorted kind of noise from the images. The image quality was measured by the RMSE and PSNR. They have yielded various hybrid filtering methods that include Anisotropic, Zernike, and Kalman filters for migration of mixed noise from MI.

\section{FA in Medical Image Registration}

The procedure of altering various sets of information into one synchronized system is known to be image registration (IR). Different types of information may include several photographs, sensors, viewpoints, times, and many more. IR can be handled in medicinal imaging, computer vision, examining and compiling images, military target detection, and information from satellites. FA in IR can be a powerful technique, due to the higher fitness value and scaling factors respectively.

Chakraborty et al. [115] has introduced a technique for recording video series that relay on demons listing based FA for optimization. The authors have used the Gaussian filter assessments for the rate leveling kernel ranging factors such as $k 1, k 2$, and $k 3$ for optimization. FA has utilized for the coefficient of link as the fitness function, so the maximum fitness value was accumulated to utilize the equivalent ranging factors for post-processing. A comparison of the proposed methodologies performance with the classic demons algorithm was made with no optimization techniques that recognized the advantage of the proposed system. Additionally, the authors have contrasted the projected FA bases model to the PSO model and found the FA contains higher scaling as well as fitness values. The work presented by Cocianu and Stan [116] was aimed at the improvement of EA to record images for the usage of signature detection. The authors have introduced and enhanced various techniques to attain fast and exact algorithms. Initially, they proposed bi variants of FA techniques that confirmed its outstanding accurateness along with fair run times. The two variant methods of accelerated particle swarm optimization (APSO) was introduced to speed up the division. Based on experimentation, the authors have got the results as the projected algorithms were found to have faster rates compared to FA based methods, yet the rates of identification was a bit lower than projected techniques. To identify the trade-off among the difficulty of calculation and 
rate of identification, the authors have improved a hybrid technique that merges the capability of the Evolution Strategies (ES) search to determine optimum resolution along with the sturdy rapid convergence capacity of APSO. The existence of angular rotation as well as translation of objects made the medicinal images as significant for the difficult post-processing methods. Consequently, medicinal image registration developed into one of the major input equipment for supporting images. The objects and images in the image registration were altered by supporting every image with a particular allusion. Demons registration is one such effective non-rigid method. Chakraborty et al. [117] has introduced methods that optimized the limitations of demons registration with FA in the retinal images. Various metrics were used for calculating the projected techniques such as mean square error (MSE), mutual information result amid the unusual and recorded images, and joint entropy (JE). Image registration has a vital part in medical imaging. Chakraborty et al. [118] has introduced grey-wolf optimizer (GWO) techniques that relay non-rigid demons listing to sustain the procedure of retinal image registration. A comparison of the projected techniques was made with FA, PSO, and CSO- based demons registration respectively. Besides, the contrastive assessment of various techniques of demons registration includes Thirion's, Tang's, and Wang's demons that are optimized with GWO. Based on the experimentation, the authors have outperformed 0.9977 as the attained correlation compared to other algorithms. The enhancement of automatic IR techniques was a renowned issue in the domain of CV. IR has been used in the massive number of current world situations that are varying from remote sensing to artificial, computer-assisted, and medical imaging design. Over the past 2 decades, the novel optimization advances were found attaining a huge interest in contracting with the major problems in IR techniques such as the ICP algorithm. Santamaría et al. [119] has performed a deep review over the last decay i.e., from 2009 to 2019 about the successful handling of optimization advances while facing the issues with IR. The authors have chosen 24 from more than 150 modern techniques. They provided various advancements by some of the particular algorithms. Based on the results, the authors have concluded that the projected techniques proved that nature-inspired algorithms have raised their attention in the IR issues over the last decades and highlighted to have further improvement.

\section{FA in Medical Image Enhancement}

Image enhancement plays a significant role in image processing. It is the procedure of emphasizing a few characteristics and is a method to enhance the data perception in images to afford superior visualization. Image enhancement uses for changing digital images to get the consequences to be appropriate for either display or analysis of an image. Medical image enhancement is having a major significance and aims the issues of low-difference and high-level noise in the exact analysis of particular illness and also for the assessment and research documentation. Draa et al. [96] has proposed OFA and applied it to medical image contrast enhancement. OFA method has been utilized to seek the better objectives of grey levels for the improvement of image contrast. The authors compared the proposed technique with FFA, AMFF (Adaptive Moment Fireflies) respectively. The proposed method was found to have a better contrast enhancement rate. They have improved the gray level images initially and then widened the color images with parallel augmentation and the projected technique has succeeded in creating a more extended histogram through the range variety of grey levels respectively. Dhal et al. [120] has introduced a new variant of histogram equalization (HE) to improve the retinal images. The authors have utilized FA, WEBTHE (weighted and entropy-based thresholded histogram equalisation) methods for the retinal images. They have utilized FA to get optimal values with different ranges by taking PSNR as the objective function. They used Retinal Image (Low Contrast) dataset for the projected method and found PSNR, Entropy, etc as performance metrics. The proposed method was compared with the ACE (automatic colour equalisation) to get better results. Based on the experiment results, it is observed that the projected technique yields better enhancement rate.

\section{FA in Medical Image Segmentation}

The procedure of separating a digital image into various sections is referred to as image segmentation (IS). IS can be utilized to set limitations and objects such as curves, lines, etc. whereas, IC can be used to build thematic maps. The popular methods of IS include edge detection, regionbased, clustering-based, watershed-based, and ANN-based techniques. Digital images have attained the utmost attention from the last few decades in the various research fields. Hrosik et al. [121] has developed an algorithm for segmentation of brain image to highlight four various brain tumors such as sarcoma, glioma metastatic bronchogenic carcinoma, and adenocarcinoma, from MRI, SPECT as well as PET images respectively. The projected image segmentation technique was relied on FA whose resolutions were enhanced by the K-means algorithm while using Otsu's principle as the fitness function. The authors have combined the projected algorithms with other images and contrasted the attained results with other techniques. It was found considering usual performance metrics that include RMSE, PSN, and SSI respectively. The process of transferring labels to a set of regions or pixels is referred as Magnetic resonance imaging segmentation. Selva Bhuvaneswari and Geetha [122] have 
introduced a segmentation and classification technique of brain images with SVM as well as FA respectively. The overall process of segmentation was detailed in three stages such as threshold making with vibrant adapted region growing stage, texture feature production stage, and section merging stage. The initial stage of the attained key image can be executed as a dynamic adapted rising process, in which the FA and optimization algorithms use to optimize the adapted regions. Here, an edge recognition algorithm was used to identify the edges. The texture feature can be extorted with entropy-based function from the key image during the second stage. The outcomes attained from the texture feature generation in the third stage were merged with the consequences of dynamic adapted raising stage respectively. After recognizing the irregular tissues, the categorization can be made with hybrid SVM. It was found using evolutionary metrics like accuracy, sensitivity as well as specificity.

\section{FA in Medical Image Detection}

The process of detecting or recognizing a feature or an object in a digital image is known to be image detection (ID). It can detect people, objects, places, and image actions. ID uses vision equipment with trained and $\mathrm{AI}$ algorithms to detect images via digital systems. Safety surveillance, toll booth supervising, and factory automation are the various applications used for image detection. FA has been prominently used in the process of image detection to identify several lesions and noisy images.

Corona virus disease (COVID-19) is one of the worldwide hazards and many dealing as well as detection procedures were advised by various examiners for COVID19. Rajinikanth et al. [123] has introduced a method with a motive of introducing an automatic image investigation system to extort and identify the Covid-19 injuries from CT scans respectively. The authors have applied some processes that include Image pre-processing to improve the COVID19, Image post-processing to extort the injuries, and implementation of a virtual analysis among the extorted and the Ground Truth Image (GTI). To execute and improve pneumonia and Markov-Random-Field (MRF) segmentation, Shannon Entropy (SE) as well as FA has been developed to extort the lesions with enhanced accurateness. The authors have concluded that the projected technique has outperformed more than $92 \%$ of the accuracy rate in the COVID19 segmentation process. FA is a dominant optimization technique that is motivated through the flashing activities of fireflies. Filipczuk et al. [124] has proposed a technique for the identification of automatic nuclei on fine needle biopsy's (FNB's) cytological images. The authors have found an issue of regular marking of nuclei on computer-assisted breast cancer analysis. Their method was relied on FNB's microscopic images. So, the recognition of malignant and benevolent lesions was the major task of their analysis. Several segmentation techniques involved a preface collection of objects on the image. So, the authors have used FA for the experimentation and also improved an initialization process. They have concluded that the outcomes proved to be satisfactorily efficient. The biggest reason behind the blindness and loss of vision for the millions of people throughout the world is diabetic retinopathy (DR). Though many screening techniques for the recognition of diseases such as optical coherence tomography and fluorescent angiography were established, many patients were continued to fail as well as unaware to commence such kinds of tests at the correct time. Several ML and DL advances have been used on DR dataset for forecasting and identification of diseases. But most of them have mistreated the data pre-processing feature and reduction of dimensionality that directed to biased outcomes. Gadekallu et al. [125] has proposed a method of hybrid principal component analysis (PCA) with FA based DNN technique for the categorization of DR dataset. The authors have improved FA for the reduction of dimensionality, and the decreased dataset was used in DNN for classification. They have concluded that the produced outcomes from the projected models have assessed against the common models of ML and the consequences have validated the advantages of proposed techniques in various aspects such as accuracy, recall, specificity, precision, and sensitivity respectively. Their advance can be utilized for removing noisy data in the analysis of Magnetoencephalography (MEG) information that is contributing to the enhanced calculation in the field of healthcare.

\section{FA in Medical Image Classification}

The process of extorting data from a multiband raster image can be referred to as image classification (IC). And, IC includes supervised, unsupervised, and object-based image analysis. FA has been used in various fields of research. Especially, FA has suitably utilized in image classification due to its convergence rate and to resolve different problems successfully. Computer-assisted diagnostics, as well as recognition techniques, are utilized to help experts in the elucidation of MI. the identification and categorization of unceasing disruptive pulmonary infection in lung $\mathrm{CT}$ images is one of the key problems in the area of research. Tuba et al. [126] has proposed a technique for emphysema categorization that relay on intensity as well as texture features. The authors have used six various intensity histograms include mean, standard deviation (SD), entropy, energy, skewness, and kurtosis, and some other consistent local binary prototype features. Based on experimentation, the authors have concluded that the projected technique has outperformed $98.18 \%$ rate of accuracy contrasted to $93.9 \%$ and $95.24 \%$ of other compared methods. A novel algorithm named Firefly RBF network was 
developed by Chao and Horng [10] to train the RBFNN by using FA for categorizing various groups of supraspinatus disease those are usual, calcific tendonitis, tendon swelling, and tears of the ultrasound supraspinatus images depends on the texture study methodology. The features of the texture were produced from four different techniques that include the grey-level co-occurrence matrix, the fractal dimension, the texture spectrum, and the method of texture feature coding to assess the tissue attribute of the supraspinatus. The projected FA-RBFNN was utilized to distinguish test images into the four compared groups of the disease in the process of classification. The authors have claimed for the best outcomes of the projected method have proved as $93.7 \%$ of correct classification rate which is more than the remaining ultrasonic supraspinatus image methods respectively.

\section{FA in image quality assessment}

The procedure of resolving the accuracy level is termed as image quality assessment (IQA). Image quality can be referred to as having various imaging structures that usually capture, accumulate, practice, broadcast, exhibit, and compact the signal to figure an image. This can be evaluated with the objective as well as subjective techniques. Image quality evolution performed by several algorithms that study the degradation and alterations of an image in the objective technique. Humans can experience or identify the quality of the image in the subjective technique. G'alvez et al. [62] has used a hybrid modified FA (HMFA) in the medical imaging (MI) for the detection of border curve of skin lesions. The authors considered convergence rate as a performance factor and compared the proposed method with uniform, chord-length as well as centripetal parameterization schemes respectively. To attain better performance, they have hybridized the modified algorithm with a local search process. Based on the experimental analysis, the proposed methods were found to attain better results for conventional mathematical models and are superior as well as competitive through accuracy. They have concluded that the projected method can be applied to the current world health applications. Malathi and Nedunchelian [127] has introduced a new method to identify blood vessels efficiently. The authors have applied the region of interest (ROI), and Kirsch's templates techniques for the extraction of retinal images as well as veins respectively. To categorize the diabetic retinopathy in fundus retina images, ANN-based FA has been applied. They considered the different factors such as the diameter of the vein and the cotton wool spot area for grouping of the concerned area. Based on the analysis, the authors have concluded that the proposed method has attained better outcomes to sustain analysis of diabetic retinopathy with precise detection.

\section{FA in Telemedicine}

Telemedicine permits healthcare (HC) to access, analyze, and cure patients at a distance by using telecommunication methodology. It is the swapping of medical data from one to another location with electric communication which enhances health updates of patients. A few examples of telemedicine include isolated health diagnosis, the digital broadcast of medical imaging, video sessions as well as assessments with specialists, and many more.

The switching of medicinal images under public networks requires a tactic to afford for the image with verification for image veracity. Swaraja [80] has proposed a hybridization of DWT along with FA based algorithm and Schur transforms with various watermarking was suggested to provide safety fundamentals that include validity of the possession of medical image as well basis of source for exchange of medical images in the applications of telemedicine. Besides the robustness of the introduced method, the eminence of the watermarked image was also enhanced with the aid of both FA and Schur. The experimental results demonstrated the performance of the projected technique that exceeds the directly connected particular value decay centered methods. The major parameters for the evolution of the watermarking algorithm were the payload, ability of imperceptibility, and robustness with ultrasound $\mathrm{x}$-ray, as well as MRI medical images. A new vigorous watermarking system that relay on FA in the transform field was developed by Sivaprakash et al. [128]. The authors have used different methods such as watermark extraction as well as embeddings that executed in the particular value disintegration and distinct wavelet transform fields respectively. They have altered the host image into a sub-band of separate frequencies in the period of embedding stage by 3-level DWT. The image of the watermark was fixed into low and high pass sub bands to change the robustness as well as the invisibility of the watermark. They have used FA to discover the optimal value and to enhance the eminence of the watermark in the medicinal image domain. Based on experimentation, the authors have got the results that notify superior invisibility contrasted to other techniques. They have concluded that the projected technique was able to attain improved performance when contrasted to other methods. Moreover, some other applications of biomedical field using FA are depicted in Table 6 .

\section{FA in Health Care}

FA has been remained a successful metaheuristic in health care domain. In the year 2018, Al-Thanoon et al. [142] has proposed a modern way of approach for establishing the parameter tuning in SCAD (Smoothly Clipped Absolute Deviation) penalty as well as PSVM using FA. This method has been applied on the selection of genes as well 


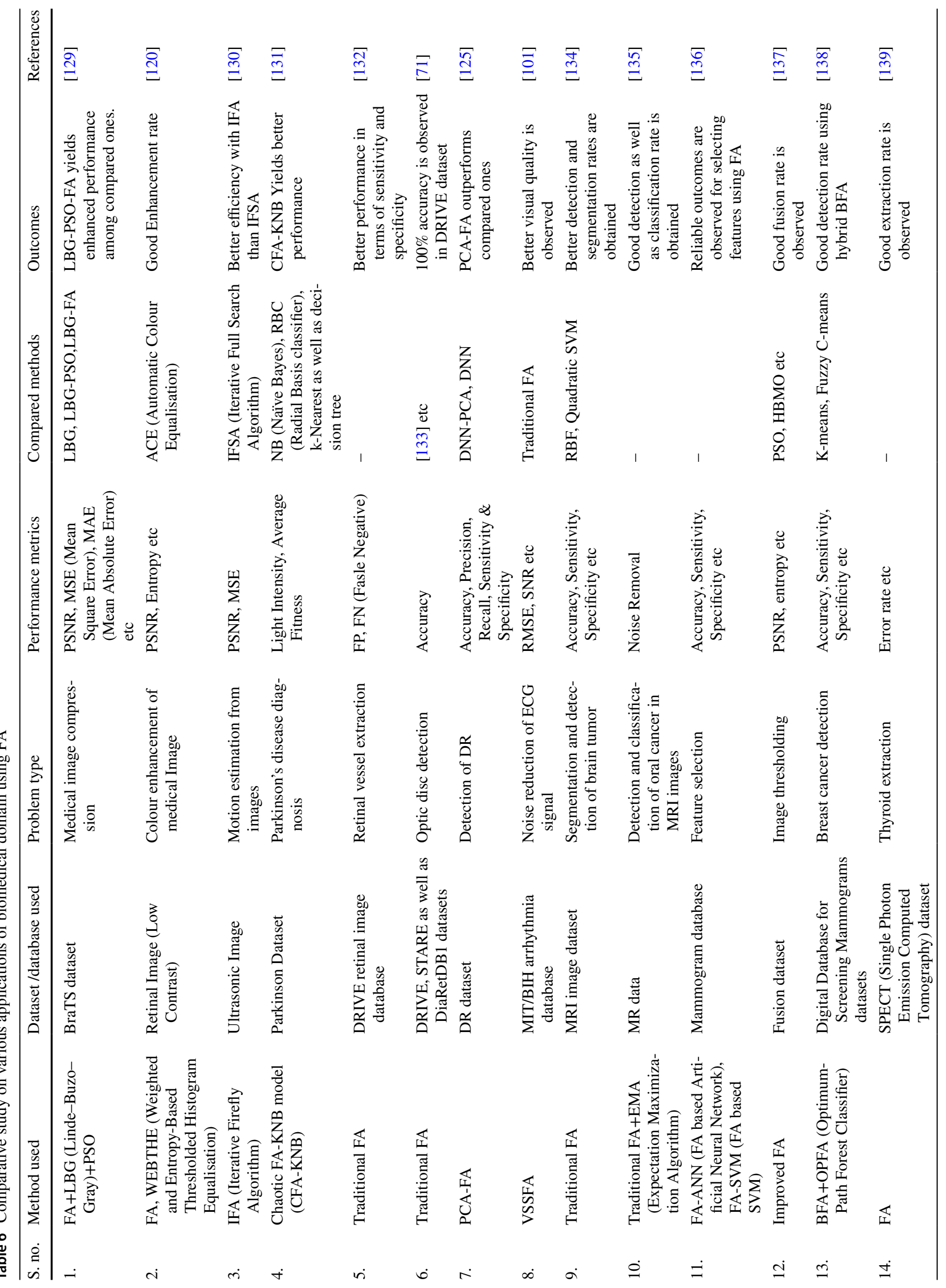




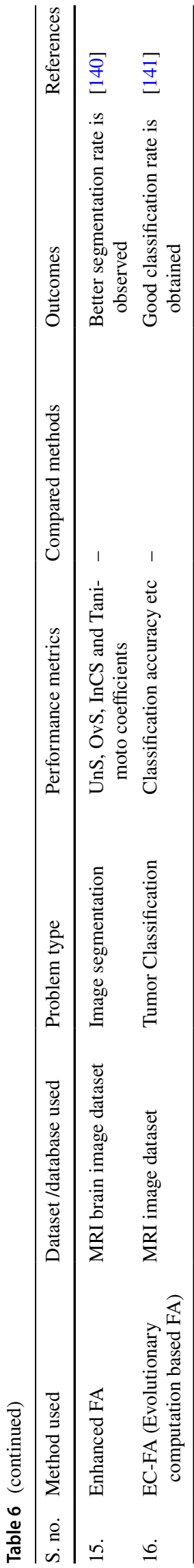

as classification of cancer. Specificity, accuracy rate etc have been taken into consideration as a performance metrics. For validating the performance, several state-of-the-art datasets such as ovarian, breast etc have been utilized and the method has been compared with some standard algorithms such as statistical CV (Cross Validation) GA, PSO and deferred better performance rather than the compared ones. Jothi and Inbarani [59] have developed a modern feature selection algorithm named Tolerance Rough Set Firefly based Quick Redacts (TRSFQR) which is the combination of FA and TRS (Tolerant Rough Set) for solving problem of classification of brain tumor from MRI images. Precision rate, recall are used as performance factors and a dataset that contains 200 MRI images have been taken into consideration for evaluating the performance. Algorithms such as ABC (Artificial Bee Colony) [143], CSA etc are compared with the proposed methodology and found that it has high capability of eradicating the features that are redundant. Also, a robust as well as consistent performance has been found from the simulated experimental results rather than the compared ones. Senapati and Dash [144] have proposed a novel way of approach for the classification of breast tumor on the basis of LLWNN (Local Linear Wavelet Neural Network) with the help of FA. FA has been utilized for optimizing the parameters of LLWNN. Wisconsin breast cancer data set has been considered for evaluating the performance and standard deviation mean has been taken into account as performance metrics. The proposed technique was later, compared with some benchmark methods such as LLS [145], SFC [146] etc and found high classification accuracy with a rate of 98.14 $\%$ for solving the classification problem of breast tumor. In the year 2018, Hashem et al. [147] has proposed a novel methodology for the classification of the jaw fracture problem, with the help of FA. Considering precision, recall as well as accuracy as performance factors and for evaluating the performance, the proposed method has been applied over benchmark datasets CT [148] etc and a comparison has been made with some state-of-the-art algorithms such as GA, PSO etc. MLPNN (Multilayered Perceptron Neural Network) has been employed to train the concerned jaw characteristics. Finally, the authors have stated that they found a better classification rate of characteristic of jaw fracture other than the compared techniques with a less error rate. Many other applications that have been utilized with FA in HC have been depicted in Table 7 .

\section{Critical Analysis}

In this research, we have conducted a systematic study on the usages and advances of the well-known metaheuristic named FA in BME \& $\mathrm{HC}$ research areas. It is practically noticeable that many SI based NIOA are applied for determining the 
Table 7 Applications of FA in $\mathrm{HC}$

\begin{tabular}{llll}
\hline S. no & FA type & Application type & References \\
\hline 1 & Hybrid FA & Myocardial Infarction detection & {$[51]$} \\
2 & FA & Heart Disease Prediction & {$[149]$} \\
3 & Hybrid FA & Clinical Decision Support System & {$[50]$} \\
4 & FA & Malaria Transmission Detection & {$[150]$} \\
5 & FA & Breast Cancer Classification & {$[151]$} \\
6 & Enhanced FA & Breast Cancer Analysis & {$[152]$} \\
7 & Hybrid FA & Gene Selection \& Cancer Classification & {$[60]$} \\
8 & Adaptive FA & Brain Tumor Detection & {$[55]$} \\
9 & FA & Construction duration of Diaphragm Wall & {$[153]$} \\
10 & FA & Lung Nodule Segmentation & {$[154]$} \\
11 & FA & Heart Disease Diagnosis & {$[155]$} \\
12 & FA & Bacteria Detection & {$[156]$} \\
13 & FA & Iris Recognition & {$[157]$} \\
14 & FA & Breast Cancer Diagnosis & {$[158]$} \\
15 & FF-SVM & Gene Selection \& Micro Array Cancer Classification & {$[159]$} \\
16 & Chaos based FA & Parkinson's Disease Diagnosis & {$[160]$} \\
17 & Hybrid FA & Biopsy Cancer Cell Segmentation & {$[161]$} \\
18 & FA & Heart Disease Diagnosis & {$[162]$} \\
\hline
\end{tabular}

\section{\% Analysis on the usage levels of BM \& HC among others related papers used in this study}

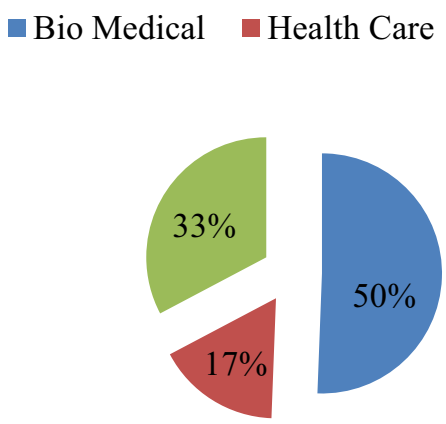

Fig. 3 Usage levels of BM \& HC over others

numerous conflicts of various application field. However in this research, it is venerated that FA is utilized to apply for resolving numerous complex problems of HC \& BME fields. The articles that are taken into consideration for this investigation are collected from the standard repositories such as IEEE Xplore, Springer, InderScience, Wiley as well as Science direct etc. The search procedure starts with the keywords search "FA in health care", "FA in biomedical field". Based on these keywords, we found a very less number of papers which is not sufficient for this research work so that we excluded these keywords and applied a new strategy (refer Sect. "Systematic Literature Review Process").

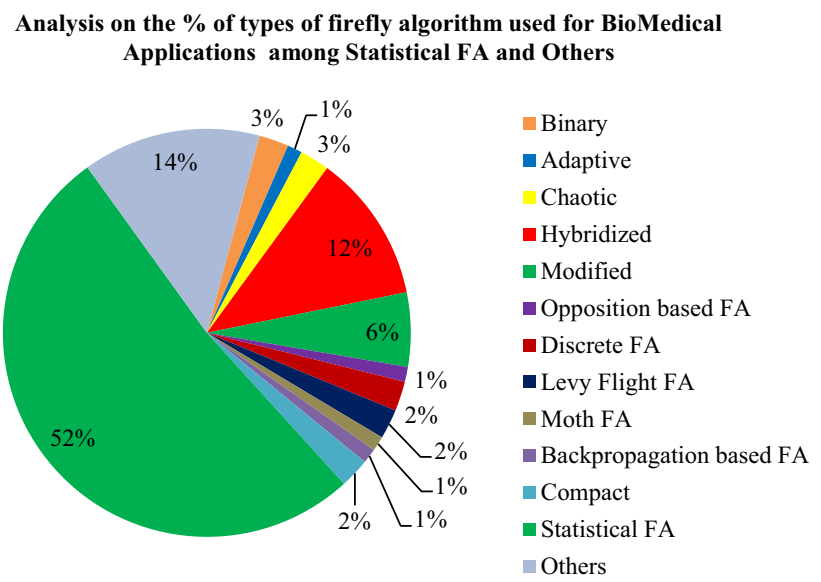

Fig. 4 Applicability of variants of FA in BME Field among Statistical FA and Others

The paper extraction strategies from several databases such as Science Direct, Springer, Wiley, IEEE Xplore, Google Scholar are depicted in Fig. 1. As our main aim of this research work is on advancements and usage levels of FA in BME as well as $\mathrm{HC}$ applications, we found a total of 111 papers related to BM \& HC. The analysis of BM \& HC over others is depicted in Fig. 3. We made an analysis on the usage levels of variations of FA in BME applications and the results obtained from the analysis have been depicted in Fig. 4. Out of 111 papers, we found that 83 papers are related to bio-medical field and from those 83 papers we found 2 chaotic, 2 Discrete FA, 2 Levy-flight FA, 1 Moth 


\section{Analysis on the \% of types of firefly algorithm used for HC among Statistical FA and Others}

Adaptive
Enhanced FA

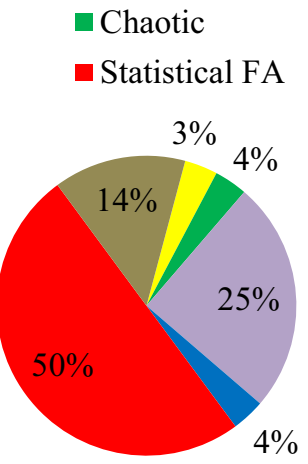

- Hybridized

- Others

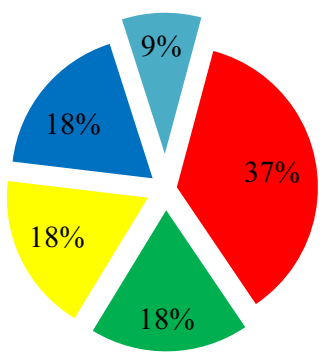

Illumination of Breast Cancer

related Problems using FA

- Breast Cancer Diagnosis

- Breast Cancer Classification

Breast Cancer Analysis

- Breast Tumor Classification

Breast Cancer Detection

Fig. $7 \%$ of utilization of FA for solving various problems of breast cancer

Fig. 5 Usage levels of FA in the application of HC among Statistical FA and others

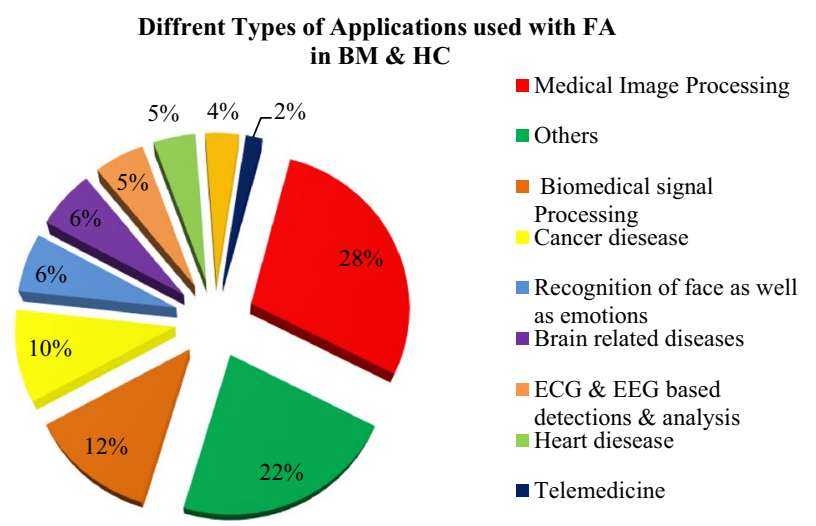

Fig. 6 Analysis on the usage levels of specific applications using FA in $\mathrm{BM}$ and $\mathrm{HC}$

FA, 1 Back propagation FA, 2 Compact FA, 2 binary variants, 1 adaptive, 5 modified, 1 opposition, and 10 hybrid variation related papers and 42 related to statistical FA and 12 others respectively.

Similarly, analysis on the usage levels of FA in HC field has been conducted and the results are depicted in Fig. 5 . Out of 111, we found that 28 papers are related to health care. From these 28 papers, we have concerned 20 papers for Table 7 and rest of the 8 for literature. Among those 28 papers, we found that there are 1 adaptive, 1 chaotic, 7 hybrid FA's, 1 Enhanced FA variations and 14 related to statistical FA and 4 for others respectively.

There is a rapid increase on the expansion of literature of FA. Several research articles are published in recent days using FA to provide the solutions for the domain of $\mathrm{HC}$ as well as BME applications. FA has been utilized in many application and surveys are conducted using specific applications such as classification [65], higher order neural

\section{Gene Selection}

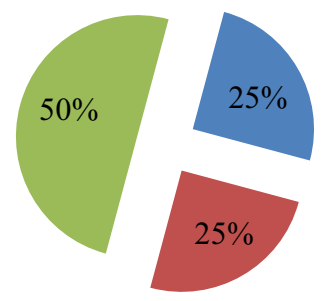

Fig. 8 Percentage of usage levels of FA for solving the problems of gene selection \& cancer classification

\section{Utilization of FA for solving Heart Related Diseases}

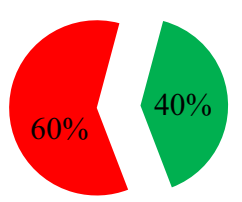

\author{
Heart Disease \\ Prediction \\ - Heart Disease \\ Diagnosis
}

Fig. 9 FA in heart disease diagnosis

networks [164], data clustering [18], image processing, robotics, wireless networks, chemistry as well as civil engineering fields etc. As per our research strategies, we found that FA has also been extensively applied in various applications of BME \& HC such as cancer classification, gene selection, heart \& brain tumor diseases prediction, recognition of speech and face, EEG \& ECG signaling, Telemedicine, Biomedical Signal Processing, Medical Image Processing and many more. All these applications are found to be 
frequently solved by the FA and hence we made an analysis on the percentage of usage levels of these frequently used applications and the results obtained from the analysis have been illustrated in Fig. 6.

We have also made in-depth analysis on each and every specific applications areas of those that were mentioned in Fig. 6. It is found that FA has been used to solve breast cancer related diseases on an average of $10 \%$. Different cancer classifications such as breast tumor classification, breast

\section{Usage of FA for resolving Brain related Issues}

- Brain Tumor Image Classification $\square$ Brain Tissue Segmentation

Brain Region Segmentation - Brain Tumour Detection

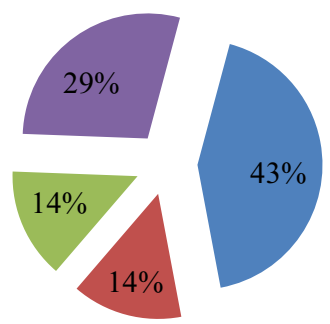

Fig. 10 Utilization of FA for solving brain disease cancer analysis, breast cancer diagnosis and breast cancer classification are the 4 different types of problems solved using FA and the usage level has been depicted in the Fig. 7 .

Similarly, analysis has been made on gene selection \& cancer related issues and the usage levels of FA for solving these types of problems are taken into concern and the usage level pattern has been illustrated in Fig. 8. From Fig. 8, it is observed that problems such as gene selection and cancer classification, gene selection and breast cancer diagnosis are combinly solved using FA.

It has been also found that the heart related problems such as heart disease prediction and heart disease diagnosis are resolved using FA and the \% of usage has been depicted in Fig. 9.

Regarding the brain related diseases, it has been found that four issues like brain tissue segmentation, brain region segmentation, brain tumor image classification and brain tumor detection issues are resolved using FA and the analysis has been depicted in Fig. 10.

Many problems has been resolved with the help of FA using medical images such as cytological image classifications, MRI image segmentations, medical image segmentations, medical image registrations, medical image contrast enhancement, MRI brain image segmentation and microscopic image segmentations etc. An analysis has been made
Fig. 11 FA in medical image processing
Usage levels of FA for solving probems using medical Images

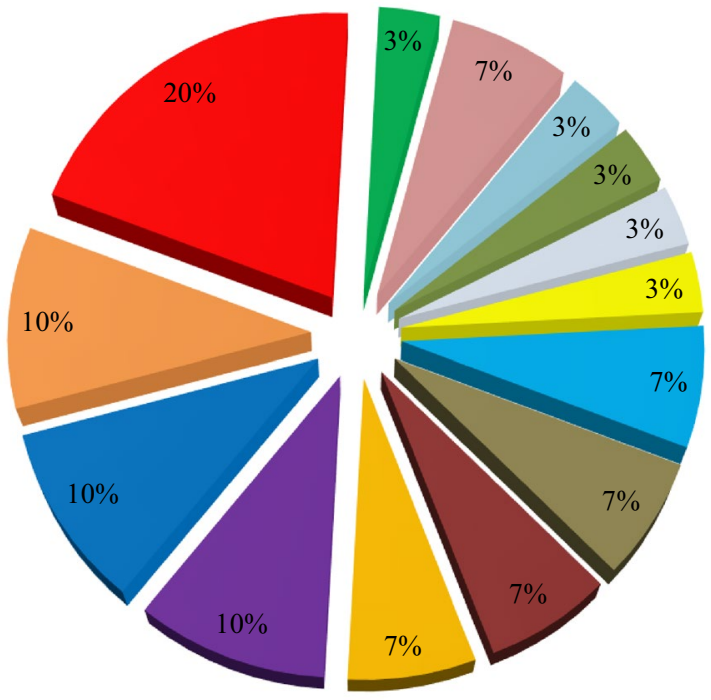

- Medical Image Thresolding 
Problems solved using FA related to various types of Recognition

- Hand Vein Recognition

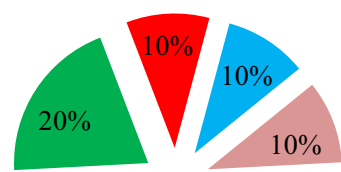

Iris Recognition

Face Recognition

- Facial Expression Recognition

Facial Emotion

Recognition

Emotion Recognition

- Speech Recognition

Fig. $12 \%$ of utilization of FA to resolve problems of different recognitions

\section{$\%$ of ECG \& EEG related issues solved using FA}

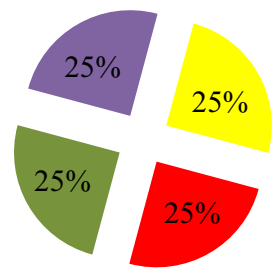

ECG based Atrial Fabiltration Detection

- EEG Signal Feature

Selection

Epilepsy Classification from EEG

- EEG Emotion Recognition

Fig. 13 Usage levels of FA for solving ECG \& EEG related problems on these problems resolution and the results have been depicted in Fig. 11.

Some of the problems related to iris recognition, face recognition, facial expressions, facial emotional recognitions, speech recognition and hand vein recognitions are also solved using FA. An analysis has also been made on these problems and the obtained outcomes have been depicted in Fig. 12.

Similarly, problems on various ECG based \& EEG based signal feature selection and atrial fibrillation \& epilepsy classifications have been solved using FA and analysis on the usage levels of these particular problems have been made and the utilization pattern has been depicted in Fig. 13.

Apart from the above mentioned problems, still there are some problems that are solved using FA such as malaria transmission determination, jaw fracture classification, health monitoring, optic disc detection, medical data classification as well as DNA protein identification, diabetes diagnosis etc. Along with these mentioned problems, some other problems are also resolved and strategy for predicting the $\%$ of utilization of FA for solving these issues has been made. The outcomes from the strategy have been depicted in Fig. 14.

Total no of papers considered in this study are 164 . Out of 164,83 papers are related to BM, 28 papers are related to $\mathrm{HC}$ and 53 papers are related to others cited for the study. Based on this data, on a scale of 1-100, an analysis has been
Fig. 14 Applicability of FA in various medical domains

\section{$\%$ of utilization of FA for solving Other problems}

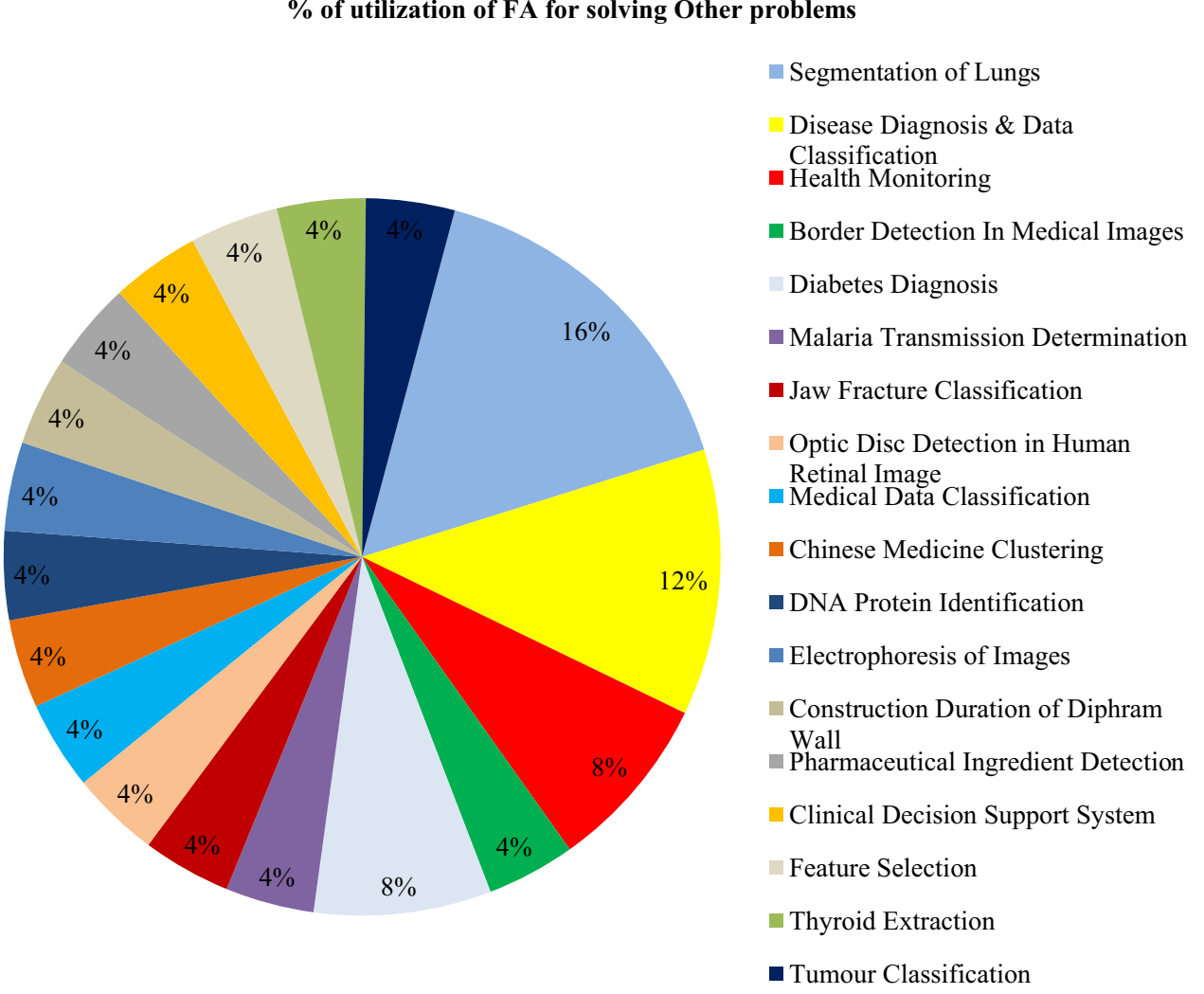


$\%$ Analysis on the usage levels of BM \& HC among Others related papers used in this study

- Bio Medical $\quad$ Health Care $\quad$ Others

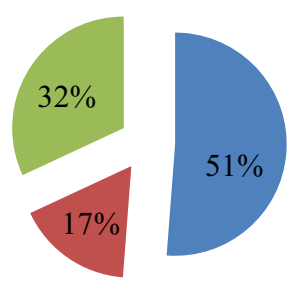

Fig. 15 Usage levels of BM \& HC among others

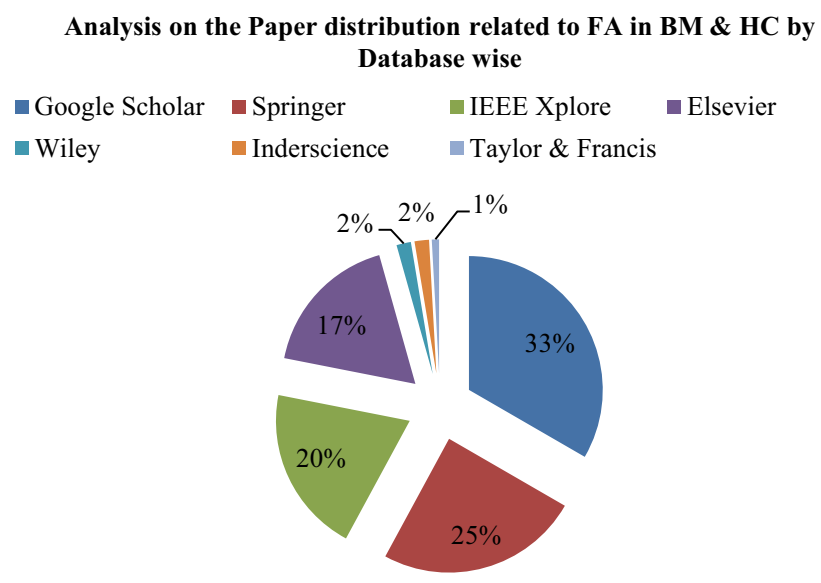

Fig. 16 Publication analysis of FA in different databases related to the considered domain (BM \& HC)

made on the usage levels of BM \& HC over others and this analysis has been depicted in Fig. 15.

Based on the keyword search applied on different databases we obtained the articles in various reputed databases related to FA in BM and HC. All these data are obtained after screening of papers using inclusion and exclusion procedures. On the basis of this data, an analysis on the usage levels of papers extracted by database wise has been made and depicted in Fig. 16.

From the data obtained for Fig. 16, an analysis has been made on the usage levels of FA related papers in BM \& HC by Journal and Conference wise. In this study, 111 papers are related to BM \& HC. Among those 111 papers, 81 papers are related to Journal and 30 are related to conference proceedings. Based on this data, an analysis has been made and depicted in Table 8 and Fig. 17.

Also, the articles have been classified by means of FA variants and its applications. On the basis of this article classification, an analysis has been made on different types of
FA used for $\mathrm{BM} \& \mathrm{HC}$ and this analysis has been mentioned in Table 9. Out of 111 considered FA in BM \& HC related articles, we have considered only some articles and from those articles, the data has been extracted and mentioned in Table 9. Based on the analysis made, it is noticeable that hybridized FA is more in number followed by Chaotic.

Likewise, year wise analysis on the distribution of papers concerned in the study has been made and the analysis has been depicted in Fig. 18. Based on the analysis, more number of articles related to BM \& $\mathrm{HC}$ is published in the year 2016. In the last decade, less number of papers related to FA in BM \& HC is published in the year 2011. From the figure, it can be analyzed that there is a significant growth in the rate of publication in the year 2016, 2018 and 2020. So, the relative publications using FA in both BM and HC is increasing with wide popularity.

Apart from all the above mentioned analysis, we found most of the researches have showed their interest towards usage of FA in BM and $\mathrm{HC}$ due to its better performance, lower computational time and faster convergence rate, etc. Tables 10 and 11 depict the performance factors used with experimentations on $\mathrm{FA}$ in $\mathrm{BM}$ and $\mathrm{HC}$ related issues.

\section{Research Challenges and Discussions}

Undoubtedly, BME \& $\mathrm{HC}$ are some of the challenging research areas and those are quite attractive towards the researchers for solving problems with the help of efficient algorithm like FA. Based on the analysis made in Sect. "Critical Analysis", it is evident that many BM \& HC dimensional related problems such as brain tissue segmentation, emotion recognition, classification of biomedical data, lung segmentation, protein identification in DNA, feature selection, health monitoring, medical image electrophoresis, medical image registration, speech recognition, medical image border detection, hand vein recognition, medical image contrast enhancement, Parkinson's disease diagnosis, biomedical signal processing, EEG epilepsy classification, EEG signal feature selection, medical image restoration, medical image preprocessing, medical image, segmentation \& classification, telemedicine, breast cancer analysis, breast cancer classification, breast cancer diagnosis, gene selection, heart disease prediction \& diagnosis, brain tissue segmentation, brain region segmentation, brain tumor detection \& classification, malaria transmission detection, diabetic detection \& diagnosis, jaw fracture classification, human optic disc detection, diaphragm wall construction, retinal vessel extraction, facial emotion \& expression recognition, iris recognition etc have been resolved by the effective use of FA. Still, it is worthy to note that there are some issues that desired to be addressed in different areas of research. FA has not yet used to cope with the problems of some applications 
Table 8 Distribution of articles related to FA in BM \& HC by Journal and Conference

\begin{tabular}{|c|c|c|c|c|}
\hline S. no & Name of Conference/Journal & Type of publication & $\begin{array}{l}\text { Frequency } \\
\text { (in number of } \\
\text { articles) }\end{array}$ & $\begin{array}{l}\text { Weight- } \\
\text { age (in } \\
\% \text { ) }\end{array}$ \\
\hline 1. & American Journal of Applied Sciences & Journal & 1 & 0.88 \\
\hline 2. & Applied Nature-Inspired Computing: Algorithms and Case Studies & Journal & 1 & 0.88 \\
\hline 3. & Applied Sciences & Journal & 2 & 1.76 \\
\hline 4. & Applied Soft Computing & Journal & 2 & 1.76 \\
\hline 5. & AUS Journal & Journal & 1 & 0.88 \\
\hline 6. & Bio-Inspired Computation and Applications in Image Processing & Journal & 1 & 0.88 \\
\hline 7. & $\begin{array}{l}\text { Biomedical Research Artificial Intelligent Techniques for biomedical signal } \\
\text { processing }\end{array}$ & Journal & 1 & 0.88 \\
\hline 8. & Biomedical Signal Processing and Control & Journal & 1 & \\
\hline 9. & BMI Bioinformatics & Journal & 1 & 0.88 \\
\hline 10. & Chemometrics and intelligent laboratory systems & Journal & 1 & 0.88 \\
\hline 11. & Chinese Journal of Integrative Medicine & Journal & 1 & 0.88 \\
\hline 12. & Classification in Bio Apps & Journal & 1 & 0.88 \\
\hline 13. & Cluster Computing & Journal & 2 & 1.76 \\
\hline 14. & Computational Mathematical Methods in Medicine & Journal & 1 & 0.88 \\
\hline 15. & Computer methods and programs in Biomedicine & Journal & 1 & 0.88 \\
\hline 16. & Computer Modeling and New Technologies & Journal & 1 & 0.88 \\
\hline 17. & Computers in Biology and Medicine & Journal & 1 & 0.88 \\
\hline 18. & Conference on Materials Science and Engineering & Journal & 1 & 0.88 \\
\hline 19. & Digital Library University of West Bohemia & Journal & 1 & 0.88 \\
\hline 20. & Electrical Engineering and Systems Science & Journal & 1 & 0.88 \\
\hline 21. & Electronics & Journal & 1 & 0.88 \\
\hline 22. & Engineering Science and Technology, an International journal & Journal & 1 & 0.88 \\
\hline 23. & Engineering Applications of Artificial Intelligence & Journal & 2 & 1.76 \\
\hline 24. & Expert systems with Applications & Journal & 1 & 0.88 \\
\hline 25. & IEEE Congress Evolutionary Computation & Conference Proceeding & 3 & 2.85 \\
\hline 26. & $\begin{array}{l}\text { IEEE International Conference on Computational Intelligence in Bioinfor- } \\
\text { matics and Computational Biology }\end{array}$ & Conference Proceeding & 1 & 0.88 \\
\hline 27. & Indian Journal of Public Health Research \& Development & Journal & 1 & 0.88 \\
\hline 28. & Information Technologies in Biomedicine & Journal & 1 & 0.88 \\
\hline 29. & Information Technology and Intelligent Transportation Systems & Journal & 1 & 0.88 \\
\hline 30. & Innovations in Electronics and Communication Engineering & Journal & 1 & 0.88 \\
\hline 31. & International Joint Conference on Neural Networks & Conference Proceeding & 1 & 0.88 \\
\hline 32. & International Conference on Computational Science and its Applications & Conference Proceeding & 1 & 0.88 \\
\hline 33. & $\begin{array}{l}\text { International Conference on Innovations in Information, Embedded and } \\
\text { Communication Systems }\end{array}$ & Conference Proceeding & 1 & 0.88 \\
\hline 34. & International Conference on Advanced Computing & Conference Proceeding & 1 & 0.88 \\
\hline 35 . & $\begin{array}{l}\text { International Conference on Application of Information and Communication } \\
\text { Technologies }\end{array}$ & Conference Proceeding & 1 & 0.88 \\
\hline 36. & $\begin{array}{l}\text { International Conference on Bio-Inspired computing: Theories and Applica- } \\
\text { tions }\end{array}$ & Conference Proceeding & 1 & 0.88 \\
\hline 37. & International conference on Biomedical Engineering & Conference Proceeding & 1 & 0.88 \\
\hline 38. & International Conference on Communication and Signal Processing & Conference Proceeding & 1 & 0.88 \\
\hline 39. & International Conference on Computational Intelligence and its applications & Conference Proceeding & 1 & 0.88 \\
\hline 40. & $\begin{array}{l}\text { International Conference on Computing, Communications and Networking } \\
\text { Technologies }\end{array}$ & Conference Proceeding & 1 & 0.88 \\
\hline 41. & International Conference on Contemporary Computing & Conference Proceeding & 2 & 1.76 \\
\hline 42. & International Conference on Control System, Computing and Engineering & Conference Proceeding & 1 & 0.88 \\
\hline
\end{tabular}


Table 8 (continued)

\begin{tabular}{|c|c|c|c|c|}
\hline S. no & Name of Conference/Journal & Type of publication & $\begin{array}{l}\text { Frequency } \\
\text { (in number of } \\
\text { articles) }\end{array}$ & $\begin{array}{l}\text { Weight- } \\
\text { age (in } \\
\%)\end{array}$ \\
\hline 43. & $\begin{array}{l}\text { International Conference on Electronics, Communication and Aerospace } \\
\text { Technology }\end{array}$ & Conference Proceeding & 1 & 0.88 \\
\hline 44. & International Conference on Hybrid Intelligent Systems & Conference Proceeding & 1 & 0.88 \\
\hline 45. & International Conference on Information and Communication Technology & Conference Proceeding & 1 & 0.88 \\
\hline 46. & $\begin{array}{l}\text { International Conference on Information Communication and Embedded } \\
\text { Systems (ICICES) }\end{array}$ & Conference Proceeding & 1 & 0.88 \\
\hline 47. & International Conference on Information Technology & Conference Proceeding & 1 & 0.88 \\
\hline 48. & $\begin{array}{l}\text { International Conference on Intelligent System Design and Engineering } \\
\text { Applications }\end{array}$ & Conference Proceeding & 1 & 0.88 \\
\hline 49. & $\begin{array}{l}\text { International Conference on ISMAC in Computational Vision and Bio } \\
\text { Engineering }\end{array}$ & Conference Proceeding & 1 & 0.88 \\
\hline 50. & $\begin{array}{l}\text { International Conference on Recent Advances in Energy-efficient Computing } \\
\text { and Communication (ICRAECC) }\end{array}$ & Conference Proceeding & 1 & 0.88 \\
\hline 51. & International Conference on Recent Trends in Image processing & Conference Proceeding & 1 & 0.88 \\
\hline 52. & International Conference on Science and Technology Computer & Conference Proceeding & 1 & 0.88 \\
\hline 53. & International Conference on Smart System, innovations and computing & Conference Proceeding & 1 & 0.88 \\
\hline 54. & $\begin{array}{l}\text { International Conference on Software, Knowledge, Information Management } \\
\text { and Applications (SKIMA) }\end{array}$ & Conference Proceeding & 1 & 0.88 \\
\hline 55. & International Conference on Swarm, Evolutionary and Memetic Computing & Conference Proceeding & 1 & 0.88 \\
\hline 56. & International Journal of Applied Engineering Research & Journal & 1 & 0.88 \\
\hline 57. & International Journal of Cognitive Informatics and Natural Intelligence & Journal & 1 & 0.88 \\
\hline 58. & International Journal of combinatorics & Journal & 1 & 0.88 \\
\hline 59. & International Journal of Computer trends and technology & Journal & 1 & 0.88 \\
\hline 60. & International Journal of Electrical and Computer Engineering & Journal & 1 & 0.88 \\
\hline 61. & International Journal of Engineering research & Journal & 1 & 0.88 \\
\hline 62. & International Journal of Image, Graphics and Signal Processing & Journal & 1 & 0.88 \\
\hline 63. & International Journal of Imaging Systems and Technology & Journal & 1 & 0.88 \\
\hline 64. & International Journal of Information and Communication Technology & Journal & 1 & 0.88 \\
\hline 65. & International Journal of Pure and Applied Mathematics & Journal & 1 & 0.88 \\
\hline 66. & International Journal of Intelligent Engineering \& Systems & Journal & 2 & 1.76 \\
\hline 67. & ISPRS Journal of Photogrammetry and Remote Sensing & Journal & 1 & 0.88 \\
\hline 68. & Journal of control engineering and applied informatics & Journal & 1 & 0.88 \\
\hline 69. & Journal of Ambient Computing and Humanized Computing & Journal & 1 & 0.88 \\
\hline 70. & Journal of Applied Sciences & Journal & 1 & 0.88 \\
\hline 71. & Journal of Biomedical Engineering and Technology & Journal & 1 & 0.88 \\
\hline 72. & Journal of Computing \& Information Systems & Journal & 1 & 0.88 \\
\hline 73. & Journal of Control Engineering and Applied Informatics & Journal & 1 & 0.88 \\
\hline 74. & Journal of Electronic Imaging & Journal & 1 & 0.88 \\
\hline 75. & Journal of Electronics and Communication Engineering & Journal & 1 & 0.88 \\
\hline 76. & Journal of Engineering and Applied Sciences & Journal & 1 & 0.88 \\
\hline 77. & Journal of Experimental \& Theoretical Artificial Intelligence & Journal & 1 & 0.88 \\
\hline 78. & Journal of Image Processing \& Pattern Recognition Progress & Journal & 1 & 0.88 \\
\hline 79. & Journal of king sand university- computer and information sciences & Journal & 1 & 0.88 \\
\hline 80. & Journal of Medical Imaging and Health Informatics & Journal & 3 & 2.65 \\
\hline 81. & Journal of Medical Systems & Journal & 1 & 0.88 \\
\hline 82. & Journal of Modern Science and Technology & Journal & 1 & 0.88 \\
\hline 83. & Journal of Visual Communication and Image Representation & Journal & 1 & 0.88 \\
\hline 84. & Knowledge and Information Systems & Journal & 1 & 0.88 \\
\hline 85. & Knowledge-Based Systems & Journal & 1 & 0.88 \\
\hline
\end{tabular}


Table 8 (continued)

\begin{tabular}{|c|c|c|c|c|}
\hline S. no & Name of Conference/Journal & Type of publication & $\begin{array}{l}\text { Frequency } \\
\text { (in number of } \\
\text { articles) }\end{array}$ & $\begin{array}{l}\text { Weight- } \\
\text { age (in } \\
\% \text { ) }\end{array}$ \\
\hline 86. & Measurement & Journal & 1 & 0.88 \\
\hline 87. & Medical \& Biological Engineering \& Computing & Journal & 1 & 0.88 \\
\hline 88. & Multidimensional Systems and Signal Processing & Journal & 1 & 0.88 \\
\hline 89. & Multimedia Tools and Applications & Journal & 2 & 1.76 \\
\hline 90. & $\begin{array}{l}\text { National Biomedical Engineering and Communications Applications Con- } \\
\text { ference }\end{array}$ & Conference Proceeding & 1 & 0.88 \\
\hline 91. & Nature Inspired Optimization Techniques for Image Processing Applications & Journal & 1 & 0.88 \\
\hline 92. & Neural Computing and Applications & Journal & 2 & 1.76 \\
\hline 93. & Neuro Computing & Journal & 1 & 0.88 \\
\hline 94. & Periodicals of Engineering and Natural Sciences & Journal & 1 & 0.88 \\
\hline 95. & Signal processing and Communications Applications Conference & Journal & 1 & 0.88 \\
\hline 96. & Structural ad Multidisciplinary optimization & Journal & 1 & 0.88 \\
\hline 97. & Structural Control Health Monitoring & Journal & 1 & 0.88 \\
\hline 98. & Swarm and Evolutionary Computation & Journal & 1 & 0.88 \\
\hline 99. & Theory and New Applications of Swarm Intelligence & Journal & 1 & 0.88 \\
\hline
\end{tabular}

$\%$ Analysis on the distribution of papers related to BM \& HC using FA by Journal and Conference wise

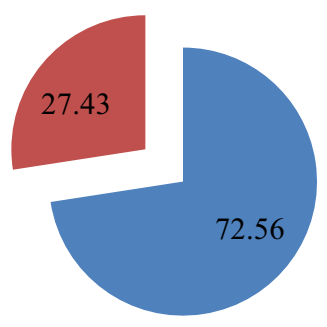

- Journal

- Conference Proceeding

Fig. 17 Usage levels of paper distribution by Journal and Conference wise

such as biomedical optics, tissue engineering (construction of artificial organs using biological material), genetic engineering (gene modification problems), pharmaceutical engineering (drug delivery), clinical engineering, biophotonics, medical informatics, hospital management, biosensors, artificial organs, biostatistics, medical robotics, biomechanics, neural engineering, rehabilitation engineering, drug discovery \& manufacturing, personalized medicine, smart health records, outbreak prediction, forecasting and clustering of medical data etc. There is a requirement to apply powerful algorithms like FA to cope with those type of application's problems that are not explored yet. In future, FA may also be explored more in these above mentioned areas of research.
It is also noticeable that only some of the variations such as binary, adaptive, chaotic, hybrid, modified, opposition based FA's have been utilized for applying in solving problems of both BM \& HC. In this scenario, still there are many numbers of variations that is needed to cope with such type of problems related to BM \& HC. It would be more fruitful to apply some variations of FA such as elitist, Gaussian, parallel and multi population etc to both BM \& HC related problems. Also, some hybridized versions of FA such as eagle strategy, genetic algorithm, differential evolution, ant colony, neural network, simulated annealing, local search, learning automata etc need to be utilized for solving the problems of BM \& HC. It is observed that some of the mentioned variants such as quaternion, eagle strategy, differential evolution, parallel and elitist based FA approaches etc have not yet used in BM \& HC field. There is a need that these variants need to be explored for solving various mentioned problems. Considering expanding the variations of FA, advance researches are needed to cope with BME, HC as well as many other domain problems.

\section{Conclusion and Future Trend}

In this study, we made a deep analysis of FA in BM and HC. FA has remained a better choice for resolving issues of two major engineering problems such as BM and $\mathrm{HC}$ due to their high elasticity in generating solutions as well as effectiveness. In the 2nd section, we detailed about FA along with its structure in Sect. "Structure of FA". We made a process of systematic literature in Sect. "Systematic Literature Review 


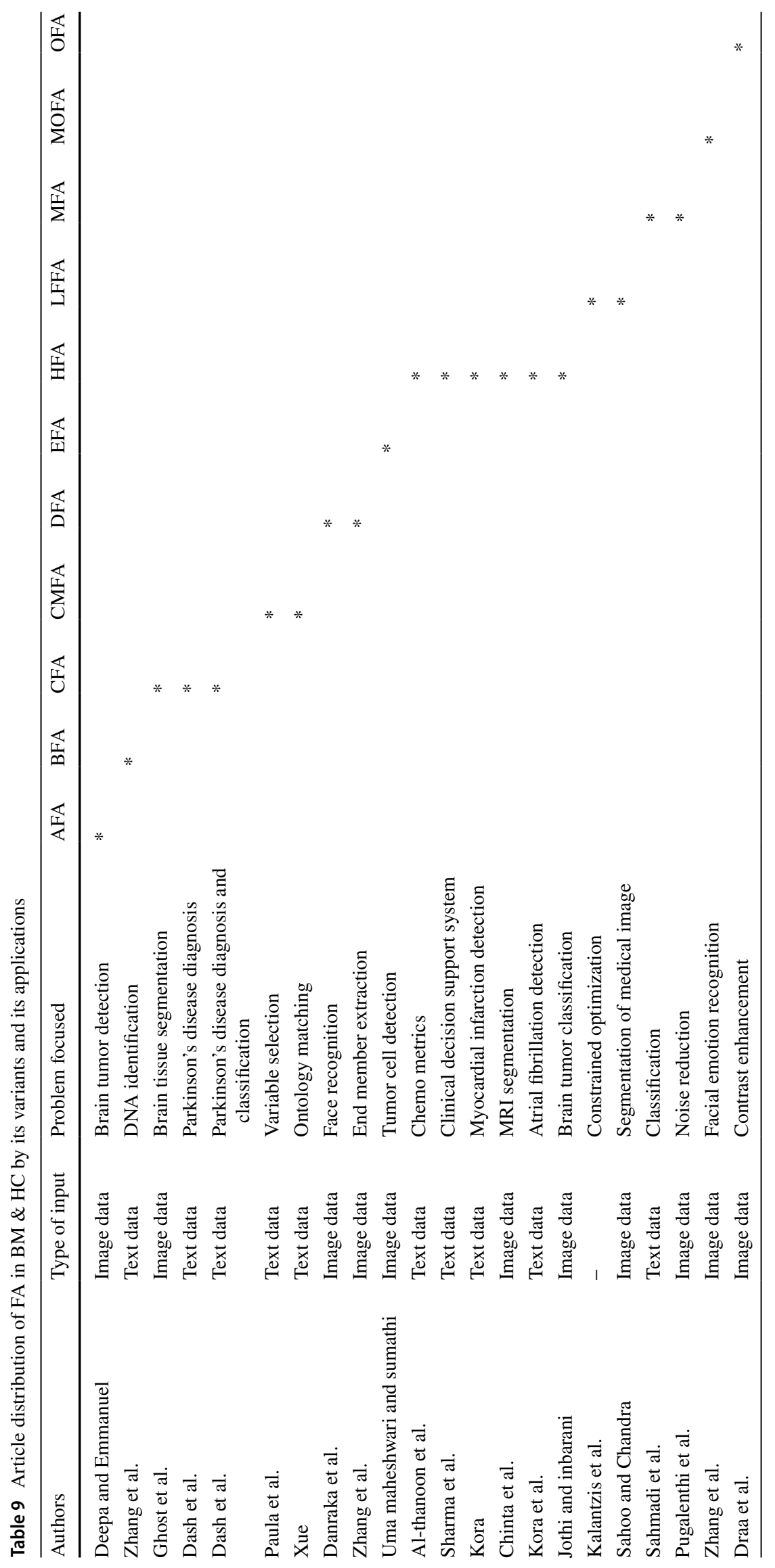


Distribution of articles of FA related to $\mathrm{BM} \& \mathrm{HC}$ by Yearwise

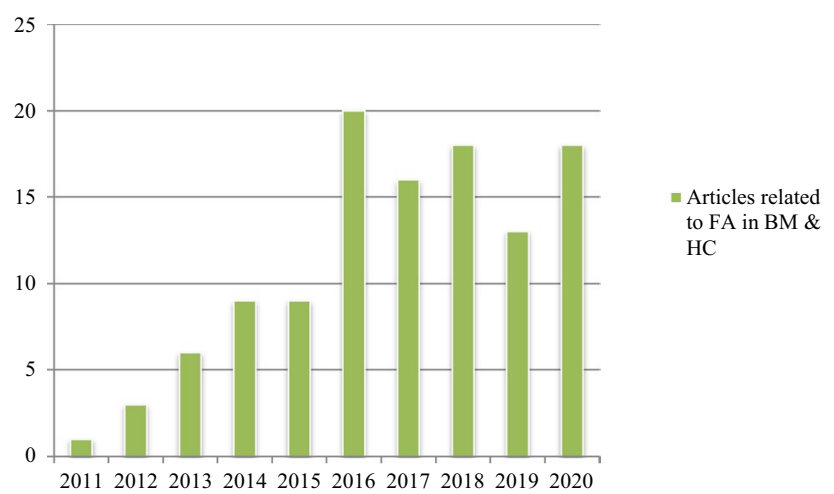

Fig. 18 Year wise analysis on paper distribution of FA in BM \& HC

Process" and inclusion as well as the exclusion criteria of papers in Sects. "Paper Inclusion Process" and "Paper Exclusion Process" respectively. In Sect. "Variants of FA used in BM and HC", we attempted to divide the variants of FA in BM and $\mathrm{HC}$ systems into five various types such as HFA, MFA, BFA, AFA as well as CFA and explained their usage in the later subsections. Later, in Sect. "Applications of FA", we turned our discussions to various applications of FA in BM and HC. Different applications include $\mathrm{BM}, \mathrm{HC}$, biomedical signal processing, IP, IR, restoration, telemedicine, and many more are discussed and detailed in subsections of applications. Section "Critical Analysis" turns the discussion into a critical investigation in which a brief study and analysis have been made to obtain an accurate assessment. We mentioned an in-depth analysis of percentage usage of BM and $\mathrm{HC}$ among other related articles, biomedical and $\mathrm{HC}$ applications amid statistical and other FA related papers, article distribution of FA in BM \& HC by Journal and Conference, year-wise analysis of FA in BM $\& \mathrm{HC}$ articles, frequently used performance metrics used with FA in BM and HC. Section "Research Challenges and

Table 10 Comparative analysis on some of the performance metrics frequently used with FA in BM and HC

\begin{tabular}{|c|c|c|c|c|c|c|c|c|}
\hline Method & Accuracy & Specificity & Sensitivity & F1 score & Precision & AUC & Recall & References \\
\hline Firefly RBF & 93.75 & 0.909 & 0.948 & NA & NA & NA & NA & [10] \\
\hline Beat features \& FFPSO & 99.3 & NA & NA & NA & NA & NA & NA & {$[22]$} \\
\hline GA-FA & 97.162 & 95.778 & 99.545 & NA & NA & NA & NA & {$[24]$} \\
\hline FFPSO & 99.3 & 98.7 & 99.97 & NA & NA & NA & NA & {$[51]$} \\
\hline DNA-binding proteins (DBPs) & 0.8989 & 0.9162 & 0.7619 & NA & NA & NA & NA & {$[53]$} \\
\hline Adaptive firefly backpropagation neural network & 99.84 & 99.85 & 97.24 & 94.26 & 98.47 & NA & NA & {$[55]$} \\
\hline Fast CS-SCHT & 99.3 & 99.43 & 96.97 & NA & NA & NA & NA & {$[58]$} \\
\hline FF-BAT & 74 & 79 & 76 & NA & NA & NA & NA & {$[63]$} \\
\hline Fuzzy Mutual Information (FMI) & 96.07 & 92.15 & 100 & NA & NA & NA & NA & {$[84]$} \\
\hline FIFS & 94.375 & NA & NA & NA & NA & NA & NA & {$[86]$} \\
\hline Tsallis+ MRF & 0.9858 & 0.9759 & 0.9957 & NA & NA & NA & NA & [95] \\
\hline $\mathrm{PSO}+\mathrm{SVM}$ & 97.21 & NA & NA & NA & 97.31 & NA & NA & [98] \\
\hline FA-KNN & 93.32 & NA & NA & NA & NA & NA & NA & [99] \\
\hline $\mathrm{MI}+\mathrm{FA}+\mathrm{SVM}$ & 95.12 & NA & NA & NA & NA & NA & NA & {$[100]$} \\
\hline FIOA & 0.8690 & 0.8300 & NA & NA & 0.7870 & NA & 0.8897 & [105] \\
\hline CTG with FA and NB & 86.54 & NA & NA & NA & NA & NA & NA & [106] \\
\hline DFA & 97.75 & NA & NA & NA & NA & NA & NA & [107] \\
\hline FFA-SVM & 0.9533 & 0.9660 & 0.9072 & 0.8934 & NA & NA & NA & [109] \\
\hline $\mathrm{CNN}$ & 92.33 & 96.2 & 84.22 & NA & NA & NA & NA & {$[110]$} \\
\hline $\mathrm{HKSVM}+\mathrm{FA}$ & 95.45 & 94.12 & 100 & NA & NA & NA & NA & [122] \\
\hline SVM-FA & 98.18 & NA & NA & NA & NA & NA & NA & [126] \\
\hline Artifact removal, FA and SE based Image processing & 98.48 & 99.49 & 90.82 & NA & 95.91 & NA & NA & {$[123]$} \\
\hline DNN-PCA Firefly & 97 & 95 & 92 & NA & 96 & NA & 96 & {$[125]$} \\
\hline CFA-KNB & 89.326 & NA & 0.893 & 0.884 & NA & 0.914 & NA & [131] \\
\hline CFARS-AR & 88.3 & 93.3 & 84.9 & NA & NA & NA & NA & [149] \\
\hline FF-BCC & $94.11 \%$ & 97.05 & 100 & NA & NA & NA & NA & [151] \\
\hline EFA and ANNIS & 92.00 & NA & NA & 94.87 & 94.87 & & 94.87 & {$[152]$} \\
\hline FSFCM & 97.56 & NA & NA & NA & NA & NA & NA & [154] \\
\hline ECFA -SVM & 97.95 & 96.50 & 97.90 & 97.90 & 97.90 & 97.20 & NA & {$[160]$} \\
\hline
\end{tabular}


Table 11 Analysis of performance factors used with FA in BM and HC

\begin{tabular}{|c|c|c|c|c|c|c|c|c|c|}
\hline Method & SNR & PSNR & RMSE & $\begin{array}{l}\text { Normalized root mean } \\
\text { square error (NRMSE) }\end{array}$ & $\begin{array}{l}\text { Noise reduction } \\
\text { accuracy (NRA) }\end{array}$ & $\begin{array}{l}\text { Gain } \\
\text { Parameter } \\
\text { (GP) }\end{array}$ & BIAS & VRMSE & References \\
\hline $\begin{array}{l}\text { Variable step size } \\
\text { firefly algorithm } \\
\text { (VSSFA) }\end{array}$ & 17.0295 & NA & 0.0963 & NA & 96.70 & 9.8723 & 0.0006 & NA & {$[101]$} \\
\hline DWT-DCT-SVD & NA & 70.8826 & NA & NA & NA & NA & NA & NA & [108] \\
\hline $\mathrm{CNN}$ & NA & 47.52 & NA & NA & NA & NA & NA & 0.77 & [110] \\
\hline Hybrid FA & NA & 18.7728 & 22.7263 & NA & NA & NA & NA & NA & [111] \\
\hline KM-FA & NA & 27.9912 & NA & 0.1559 & NA & NA & NA & NA & [121] \\
\hline FA & NA & 33.5432 & 28.56 & NA & NA & NA & NA & NA & [129] \\
\hline
\end{tabular}

Discussions" turns the discussion to research challenges for FA in BM and $\mathrm{HC}$.

The progress of computer and network methodologies has carried us various promising ways to build a useful information system for our routine life. In the same way, BM and $\mathrm{HC}$ have also made better progress in modern years. In general, metaheuristic can be considered as part of soft computing (SC) as well as ML methodologies. Besides input and output, the alteration, assessment, and determination functions will be executed frequently until the search procedure congregates or assembles the predefined ending situation became the major feature of metaheuristic. Based on our examination, we found various modern investigations were made on BM and $\mathrm{HC}$ systems with FA based metaheuristic on a larger extent. Some metaheuristic algorithms such as ACO, PSO, FA, and GA, etc can be used effectively for resolving issues of $\mathrm{HC}$ and $\mathrm{BM}$ to afford a better result. FA is one of the most popular metaheuristic algorithms for healthcare. FA has broadly extended its vicinity of application since its development. Now-a-days, the firefly algorithm has been apprehensive almost in all areas respectively. Besides, increased applications of FA are extremely vigorous, as new applications are improving each day. This investigative study is obvious that FA is appropriate and fit for resolving BM and $\mathrm{HC}$ domain problems. Medical databases are one of the major points of concern for getting a huge amount of information. FA in BM engineering and $\mathrm{HC}$ played a vital role in generating enormous solutions. Studying of early research, those are extorted from standard repositories evident that FA and its modified developments are served as a contrivance for $\mathrm{HC}$ and $\mathrm{BM}$ applications. Contrasted to the rulebased as well as deterministic algorithms, FA in HC and BM generally can offer a better outcome. Because FA is capable to present an estimated solution with less computation instance while contrasted with remaining algorithms, they have turned out to be a promising study trend of BM and $\mathrm{HC}$ in recent years, particularly for the methods that require getting consequences at valid time. Here, we have mentioned regularly used types of bio-medical engineering and healthcare problems using FA such as breast cancer classification, breast tumor classification, detection of brain tumor and health monitoring, and many more along with the investigation of the research popularity of FA in $\mathrm{HC}$ and BME in-depth manner.

Research challenges like tissue engineering, pharmaceutical engineering, information fusion, data analytics system, knowledge interpretation, security, and privacy yet to be explored. Till the day, combining and representing of extorted data from various resources of HC systems is a complex task. So, researchers require considering the problems of data fusion when they desire to enhance and execute an affective HC system. Similarly, major researchers have attracted the categorization of data analytics due to having a large quantity of hidden data. But still, there is a requirement of considering problems on data analytics, majorly the accurateness rate of information analytics. Security and privacy are one more important study trend. As overall information of $\mathrm{HC}$ and $\mathrm{BM}$ are centralized in a particular scheme to construct it feasible for the organization to assess this information with a complete view. However, the protection of this huge amount of information has become a noteworthy study challenge for the researchers. So, utilization of metaheuristic like FA to defend the HC information is one of the major explore trends that need to be considered in the coming future. There is a quick chance of enlarging using FA that can persuade advanced research directions in the environs of perspective and it may be a concern of future research. It can't be overstated to conclude that, the improvements with FA and its varieties are to be commenced and urbanized for resolving complex problems in forthcoming days.

\section{Compliance with Ethical Standards}

Conflict of interest The authors declare that this manuscript has no conflict of interest with any other published source and has not been published previously (partly or in full). No data have been fabricated or manipulated to support our conclusions. 


\section{References}

1. Storn R, Price K. Differential evolution-a simple and efficient heuristic for global optimization over continuous spaces. J Global Optim. 1997;11(4):341-59.

2. Goldberg D, Holland J. Genetic algorithms and machine learning. Mach Learn. 1988;3(2):95-9.

3. Rajasekhar A, et al. Computing with the collective intelligence of honey bees-a survey. Swarm Evolution Comput. 2017;32:25-48.

4. Dorigo M, Stützle T. The ant colony optimization metaheuristic: algorithms, applications, and advances. Handbook of metaheuristics. Boston: Springer; 2003. p. 250-85.

5. Kennedy J, Eberhart RC. Particle swarm optimization proceeding IEEE international conference of neural network IV. In: IEEE Service centre, Piscataway. 1995.

6. Zhao S-Z, Ponnuthurai NS, Swagatam D. Dynamic multi-swarm particle swarm optimizer with sub-regional harmony search. In: IEEE Congress on Evolutionary Computation. IEEE, 2010.

7. Yang X-S, Deb S. Cuckoo search: recent advances and applications. Neural Comput Appl. 2014;24(1):169-74.

8. Yang X-S. Firefly algorithm, stochastic test functions and design optimisation. Int J Bio-inspired Comput. 2010;2(2):78-84.

9. Del Ser J, et al. Bio-inspired computation: where we stand and what's next. Swarm Evolution Comput. 2019;48:220-50.

10. Chao C-F, Horng M. Firefly algorithm for training the radial basis function network in ultrasonic supraspinatus image classification. Comput Model New Technol. 2014;18.3:77-83.

11. Horng $\mathrm{M}-\mathrm{H}$. Vector quantization using the firefly algorithm for image compression. Expert Syst Appl. 2012;39(1):1078-91.

12. Banati H, Bajaj M. Fire fly based feature selection approach. Int J Comput Sci Issues (IJCSI). 2011;8(4):473.

13. Chatterjee A, Gautam KM, Arindam C. Design of a fully digital controlled reconfigurable switched beam concentric ring array antenna using firefly and particle swarm optimization algorithm. Progress Electromagn Res. 2012;36:113-31.

14. Apostolopoulos T, Vlachos A (2010) Application of the firefly algorithm for solving the economic emissions load dispatch problem. Int J Combinator. 2011:523806. https://doi. org/10.1155/2011/523806

15. Reddy KS, Reddy MD. Economic load dispatch using firefly algorithm. Int J Eng Res Appl. 2012;24:2325-30.

16. Jati GK. Evolutionary discrete firefly algorithm for travelling salesman problem. In: International conference on adaptive and intelligent systems. Springer, Berlin, Heidelberg, 2011.

17. Karthikeyan S, et al. A hybrid discrete firefly algorithm for solving multi-objective flexible job shop scheduling problems. Int J Bio-Inspired Comput. 2015;7(6):386-401

18. Senthilnath J, Omkar SN, Mani V. Clustering using firefly algorithm: performance study. Swarm Evolution Comput. 2011;1(3):164-71.

19. Nandy S, Partha PS, Achintya D. Analysis of a nature inspired firefly algorithm based back-propagation neural network training. arXiv preprint arXiv: 1206.5360; 2012.

20. Alb M, et al. Firefly algorithm for finding optimal shapes of electromagnetic devices. IEEE Trans Magnet. 2015;52(3):1-4.

21. Chandrasekaran K, Sishaj PS. Optimal deviation based firefly algorithm tuned fuzzy design for multi-objective UCP. IEEE Trans Power Syst. 2012;28.1:460-71.

22. Tsai C-W et al (2016) Metaheuristic algorithms for healthcare: open issues and challenges. Comput Electr Eng. 53:421-434. https://doi.org/10.1016/j.compeleceng.2016.03.005.

23. Veeramuthu A, Meenakshi S. Breeding firefly association rules for effective medical image retrieval. Biomedical Res Artif Intell Tech Bio Med Signal Process. 2017.
24. Mazen F, Rania AAS, Amr MG. Genetic algorithm and firefly algorithm in a hybrid approach for breast cancer diagnosis. Int $\mathbf{J}$ Comput Trends Technol (IJCTT). 2016;322:62-8.

25. Nandhini M, Rajalakshmi M, Sivanandam SN. Experimental and statistical analysis on the performance of firefly based predictive association rule classifier for health care data diagnosis. J Control Eng Appl Inf. 2017;19(2):101-10.

26. Yang X-S. Nature-inspired metaheuristic algorithms. Luniver press. Beckington, UK; 2008, pp. 242-246.

27. Yang X-S. Firefly algorithms for multimodal optimization. International symposium on stochastic algorithms. Berlin, Heidelberg: Springer; 2009.

28. Yang X-S, He X. Firefly algorithm: recent advances and applications. Int J Swarm Intell. 2013;1(1):36-50.

29. Wolpert D, Macready W. No free lunch theorems for optimization. IEEE Trans Evol Comput. 1997;1(1):67-82.

30. Saraç E, Selma AÖ. Web page classification using firefly optimization. In: Innovations in intelligent systems and applications (INISTA), 2013 IEEE International Symposium. 2013.

31. Shafaati M, Mojallali $\mathrm{H}$. Modified firefly optimization for IIR system identification. J Control Eng Appl Inf. 2012;14(4):59-69.

32. Shakarami MR, Reza S. A new approach for network reconfiguration problem in order to deviation bus voltage minimization with regard to probabilistic load model and DGs. Int J Electr Comput Energ Electr Commun Eng. 2014;8.2:430-5.

33. Yu S, Yang S, Su S (2013) Self-adaptive step firefly algorithm. J Appl Math. https://doi.org/10.1155/2013/832718.

34. Yu S, et al. A novel wise step strategy for firefly algorithm. Int J Comput Math. 2014;91.12:2507-13.

35. Lin X, Zhong Y, Zhang H. An enhanced firefly algorithm for function optimisation problems. Int J Model Ident Control. 2013;18(2):166-73.

36. Tilahun S, Ong HC. Modified firefly algorithm. J Appl Math. 2012;467631:11.

37. Gandomi AH, et al. Firefly algorithm with chaos. Commun Nonlinear Sci Numer Simul. 2013;18.1:89-98.

38. Khalil A. Improved Firefly algorithm for unconstrained optimization problems. Int J Comput Appl Technol Res. 2014;4(1):77-81.

39. Yan X, et al. An improved firefly algorithm with adaptive strategies. Adv Sci Lett. 2012;16.1:249-54.

40. Sulaiman MH, Hamdan D, Mohd WM. Modified firefly algorithm in solving economic dispatch problems with practical constraints. In: 2012 IEEE International Conference on Power and Energy (PECon). IEEE; 2012.

41. Wang B, et al. A modified firefly algorithm based on light intensity difference. J Combinat Optimiz. 2016;31.3:1045-60.

42. Othman M, Hegazy Y, Abdelaziz A. A modified firefly algorithm for optimal sizing and siting of voltage controlled distributed generators in distribution networks. Period Polytech Electr Eng Comput Sci. 2015;59(3):104-9.

43. Subramanian R, Thanushkodi K. An efficient firefly algorithm to solve economic dispatch problems. Int J Soft Comput Eng. 2013;2(1):52-5.

44. Liu C et al (2015) Three-dimensional path planning method for autonomous underwater vehicle based on modified firefly algorithm. Math Probl Eng. https://doi.org/10.1155/2015/561394.

45. Fu Q, et al. A novel firefly algorithm based on improved learning mechanism. In: International conference on logistics engineering, management and computer science (LEMCS 2015). Atlantis Press; 2015.

46. Fister I, Xin-She Y, Janez B. Memetic self-adaptive firefly algorithm. Swarm Intell Bio-inspired Comput Elsevier. 2013;2013:73-102.

47. dos Santos CL, Viviana CM. Improved firefly algorithm approach applied to chiller loading for energy conservation. Energy Build. 2013;59:273-8. 
48. Ghosh P, Kalyani M, Sitansu KD. Chaotic firefly algorithm-based fuzzy C-means algorithm for segmentation of brain tissues in magnetic resonance images. J Vis Commun Image Represent. 2018;54:63-79.

49. Al-Thanoon NA, Omar SQ, Zakariya YA. A new hybrid firefly algorithm and particle swarm optimization for tuning parameter estimation in penalized support vector machine with application in chemometrics. Chemometrics Intell Lab Syst. 2019;184:142-152.

50. Sharma M, Singh G, Singh R (2018) Clinical decision support system query optimizer using hybrid Firefly and controlled Genetic Algorithm. J King Saud Univ-Comput Inf Sci. https:// doi.org/10.1016/j.jksuci.2018.06.007.

51. Kora P. ECG based myocardial infarction detection using hybrid firefly algorithm. Comput Methods Programs Biomed. 2017;152:141-8.

52. Chinta SS, Abhay J, Tripathy BK. Image segmentation using hybridized firefly algorithm and intuitionistic fuzzy C-Means. In: Proceedings of first international conference on smart system, innovations and computing. Springer, Singapore; 2018.

53. Zhang $\mathrm{J}$, et al. Identification of DNA-binding proteins using multi-features fusion and binary firefly optimization algorithm. BMC Bioinform. 2016;171:323.

54. Sahmadi B et al. A modified firefly algorithm with support vector machine for medical data classification. In: IFIP international conference on computational intelligence and its applications. Springer, Cham; 2018.

55. Deepa AR, Emmanuel WRS. An efficient detection of brain tumor using fused feature adaptive firefly backpropagation neural network. Multimedia Tools Appl. 2019;78.9:11799-814.

56. Hassanzadeh T, Karim F, Golnaz S. A speech recognition system based on structure equivalent fuzzy neural network trained by firefly algorithm. In: 2012 international conference on biomedical engineering (ICOBE). IEEE; 2012.

57. Wang J, et al. Improvement and application of hybrid firefly algorithm. IEEE Access. 2019;7:165458-165477.

58. Kora P, et al. ECG based atrial fibrillation detection using sequence ordered complex Hadamard transform and hybrid firefly algorithm. Eng Sci Technol Int J. 2017;20.3:1084-91.

59. Jothi G. Hybrid Tolerance Rough Set-Firefly based supervised feature selection for MRI brain tumor image classification. Appl Soft Comput. 2016;46:639-51.

60. Srivastava A, et al. Hybrid firefly based simultaneous gene selection and cancer classification using support vector machines and random forests. In: Proceedings of seventh international conference on bio-inspired computing: theories and applications (BICTA 2012). Springer, India; 2013.

61. Jayalakshmi V, Rao ARM. Simultaneous identification of damage and input dynamic force on the structure for structural health monitoring. Struct Multidiscipl Optimiz. 2017;55.6:2211-38.

62. Gálvez A et al. Hybrid modified firefly algorithm for border detection of skin lesions in medical imaging. In: 2019 IEEE congress on evolutionary computation (CEC). IEEE, 2019.

63. Reddy GT, Neelu K. Hybrid firefly-bat optimized fuzzy artificial neural network based classifier for diabetes diagnosis. Int J Intell Eng Syst. 2017;10.4:18-27.

64. Zhou G-D, et al. Energy-aware wireless sensor placement in structural health monitoring using hybrid discrete firefly algorithm. Struct Control Health Monit. 2015;22.4:648-66.

65. Nayak J et al. applications and advancements of firefly algorithm in classification: an analytical perspective. In: Computational intelligence in pattern recognition. Springer, Singapore; 2020. pp. 1011-1028.

66. Nekkaa M, Boughaci D. A memetic algorithm with support vector machine for feature selection and classification. Memetic Comput. 2015;7.1:59-73.
67. Nekkaa M, Boughaci D. Hybrid harmony search combined with stochastic local search for feature selection. Neural Process Lett. 2016;44(1):199-220.

68. Yuan F, et al. A clustering method of Chinese medicine prescriptions based on modified firefly algorithm. Chin J Integr Med. 2016;22.12:941-6.

69. Sánchez D, Melin P, Castillo O. Optimization of modular granular neural networks using a firefly algorithm for human recognition. Eng Appl Artif Intell. 2017;64:172-86.

70. Krawczyk B, Filipczuk Paweł. Cytological image analysis with firefly nuclei detection and hybrid one-class classification decomposition. Eng Appl Artif Intell. 2014;31:126-35.

71. Rahebi Javad, Hardalaç Fırat. A new approach to optic disc detection in human retinal images using the firefly algorithm. Med Biol Eng Compu. 2016;54(2-3):453-61.

72. Sawhney R, Puneet M, Ravi S. A firefly algorithm based wrapper-penalty feature selection method for cancer diagnosis. In: International conference on computational science and its applications. Springer, Cham; 2018.

73. Kalantzis G, et al. Investigations of a GPU-based levy-firefly algorithm for constrained optimization of radiation therapy treatment planning. Swarm Evolut Comput. 2016;26:191-201.

74. Zhang L, et al. Intelligent facial emotion recognition using mothfirefly optimization. Knowl-Based Syst. 2016;111:248-67.

75. Khennak I, Drias H. A firefly algorithm-based approach for pseudo-relevance feedback: Application to medical database. J Med Syst. 2016;40(11):240.

76. Raja N, Madhava S, Visali-Lakshmi PR, Gunasekaran KP. Firefly algorithm-assisted segmentation of brain regions using Tsallis entropy and Markov random field. In: Innovations in electronics and communication engineering. Springer, Singapore, 2018. pp. 229-237.

77. Jangam E, Rao ACS. Segmentation of lungs from chest $X$ rays using firefly optimized fuzzy C-means and level set algorithm. In: International conference on recent trends in image processing and pattern recognition. Springer, Singapore; 2018.

78. Kumar SN, et al. Firefly optimization based improved fuzzy clustering for CT/MR image segmentation. Nature inspired optimization techniques for image processing applications. Cham: Springer; 2019. p. 1-28.

79. Bhattacharyya S, et al. Feature selection of motor imagery EEG signals using firefly temporal difference Q-Learning and support vector machine. In: International conference on swarm, evolutionary, and memetic computing. Springer, Cham; 2013.

80. Swaraja K. Medical image region based watermarking for secured telemedicine. Multimedia Tools Appl. 2018;77(21):28249-80.

81. Sahoo A, Satish C. L'evy-flight firefly algorithm based active contour model for medical image segmentation. In: 2013 sixth international conference on contemporary computing (IC3). IEEE; 2013.

82. Noor MHM, et al. Multilevel thresholding of gel electrophoresis images using firefly algorithm. In: 2011 IEEE international conference on control system, computing and engineering. IEEE, 2011.

83. Damayanti A, Asri BP. Epilepsy detection on EEG data using backpropagation, firefly algorithm and simulated annealing. In: 2016 2nd international conference on science and technologycomputer (ICST). IEEE; 2016.

84. Rajaguru H, Sunil KP. Fuzzy mutual information and firefly algorithm for epilepsy classification from EEG. In: 2018 second international conference on electronics, communication and aerospace technology (ICECA). IEEE; 2018.

85. Xiaogang D, et al. An algorithm multi-resolution medical image registration based on firefly algorithm and Powell. In: 2013 third international conference on intelligent system design and engineering applications. IEEE; 2013. 
86. Agarwal V, Surekha B. Firefly inspired feature selection for face recognition. In: 2015 Eighth international conference on contemporary computing (IC3). IEEE; 2015.

87. Preethi J, Sowmiya S. Emotion recognition from EEG signal using ISO-FLANN with firefly algorithm. In: 2016 international conference on communication and signal processing (ICCSP). IEEE; 2016.

88. Mistry K, et al. Facial expression recognition using firefly-based feature optimization. In: 2017 IEEE congress on evolutionary computation (CEC). IEEE; 2017.

89. Nuha HH, Abido M. Firefly algorithm for log-likelihood optimization problem on speech recognition. In: 2016 4th international conference on information and communication technology (ICoICT). IEEE; 2016.

90. de Paula LCM, et al. A compact firefly algorithm for the variable selection problem in pharmaceutical ingredient determination. In: 2016 IEEE congress on evolutionary computation (CEC). IEEE; 2016.

91. Alsmadi MutasemK. A hybrid firefly algorithm with fuzzy-C mean algorithm for MRI brain segmentation. Am J Appl Sci. 2014;11(9):1676-91.

92. Honarpisheh Z, Karim F. An efficient dorsal hand vein recognition based on firefly algorithm. Int J Electr Comput Eng. 2013;3.1:2088-8708.

93. Alomoush WK, et al. Segmentation of MRI brain images using FCM improved by firefly algorithms. J Appl Sci. 2014;14.1:66-71.

94. Horng M-H, et al. Firefly metaheuristic algorithm for training the radial basis function network for data classification and disease diagnosis. Theory New Appl Swarm Intell. 2012;4.7:115-32.

95. Rajinikanth V, Raja NSM, Kamalanand K. Firefly algorithm assisted segmentation of tumor from brain MRI using Tsallis function and Markov random field. J Control Eng Appl Inf. 2017;193:97-106.

96. Draa A, Zeyneb B. Fatima ZD An opposition-based firefly algorithm for medical image contrast enhancement. Int J Inf Commun Technol. 2015;7.4-5:385-405.

97. Xue X. A compact firefly algorithm for matching biomedical ontologies. Knowl Inf Syst. 2020;2020:1-17.

98. Kumar R. Optimized feature selection for the classification of uterine magnetomyography signals for the detection of term delivery. Biomed Signal Process Control. 2020;58:101880.

99. Ali NJ, Abed JK. An integrated firefly algorithm with K-nearest neighbor for cardiotocography classification. 2019. https://doi. org/10.4206/aus.2019.n26-60.

100. Jabar SF. A classification model on tumor cancer disease based mutual information and firefly algorithm. Period Eng Natural Sci. 2019;7(3):1152-62.

101. Sundararaj V. An efficient threshold prediction scheme for wavelet based ECG signal noise reduction using variable step size firefly algorithm. Int J Intell Eng Syst. 2016;9(3):117-26.

102. Akhavan-Amjadi M. Fetal electrocardiogram modeling using hybrid evolutionary firefly algorithm and extreme learning machine. Multidimension Syst Signal Process. 2020;31(1):117-33.

103. Gálvez A, et al. Firefly algorithm approach for rational Bézier border reconstruction of skin lesions from macroscopic medical images.In: 2019 13th international conference on software, knowledge, information management and applications (SKIMA). IEEE, 2019.

104. El_Tokhy MS. Ultimate neutron and x-ray radiography images compression using artificial bee colony and firefly optimization algorithms. J Electron Imaging. 2020;292:023003.

105. He H, et al. Strengthen EEG-based emotion recognition using firefly integrated optimization algorithm. Appl Soft Comput. 2020;2020:106426.
106. Kadhim NJA, Jameel KA. Enhancing the prediction accuracy for cardiotocography (CTG) using firefly algorithm and naive Bayesian classifier. MS\&E. 2020;745.1:012101.

107. Danraka SS, et al. Discrete firefly algorithm based feature selection scheme for improved face recognition. Comput Inf Syst. 2019;232:23-34.

108. Dey N, et al. Firefly algorithm for optimization of scaling factors during embedding of manifold medical information: an application in ophthalmology imaging. J Med Imaging Health Inf. 2014;43:384-94.

109. Keerthiveena B, et al. Computer-aided diagnosis for diabetic retinopathy based on firefly algorithm. In: 2019 11th international conference on advanced computing (ICoAC). IEEE; 2019.

110. Elhoseny M, Shankar K. Optimal bilateral filter and convolutional neural network based denoising method of medical image measurements. Measurement 2019;143:125-135.

111. Sam BB, Lenin Fred A. Denoising medical images using hybrid filter with firefly algorithm. In: 2019 international conference on recent advances in energy-efficient computing and communication (ICRAECC). IEEE; 2019.

112. Pugalenthi R, Sheryl Oliver A, Anuradha M (2020) Impulse noise reduction using hybrid neuro-fuzzy filter with improved firefly algorithm from X-ray bio-images. Int J Imaging Syst Technol. https://doi.org/10.1002/ima.22453.

113. Zhang $\mathrm{C}$, et al. Endmember extraction from hyperspectral image based on discrete firefly algorithm (EE-DFA). ISPRS J Photogram Remote Sens. 2017;126:108-119.

114. Csam BB, et al. A survey on image restoration using hybrid channel based on firefly algorithm. In: 2017 international conference on information communication and embedded systems (ICICES). IEEE; 2017.

115. Chakraborty S, et al. Firefly algorithm for optimized nonrigid demons registration. In: Bio-inspired computation and applications in image processing. Academic Press, 2016. pp. 221-237.

116. Cocianu C-L, Stan A. New evolutionary-based techniques for image registration. Appl Sci. 2019;9(1):176.

117. Chakraborty S, et al. Optimized Tang's Algorithm for retinal image registration. Inf Technol Intell Transport Syst. 2020;323:142.

118. Chakraborty S, et al. Grey-Wolf-Based Wang's Demons for retinal image registration. Entropy. 2020;226:659.

119. Santamaría J, et al. An overview on the latest nature-inspired and metaheuristics-based image registration algorithms. Appl Sci. 2020;10(6): 1928.

120. Dhal KG, Sanjoy D. Colour retinal images enhancement using modified histogram equalisation methods and firefly algorithm. Int J Biomed Eng Technol 2018;28.2:160-184.

121. Hrosik F, Capor R, et al. Brain image segmentation based on firefly algorithm combined with k-means clustering. Stud Inform Control. 2019;28:167-176.

122. Selva BK, Geetha P. Segmentation and classification of brain images using firefly and hybrid kernel-based support vector machine. J Experim Theor Artif Intell. 2017;29.3:663-678.

123. Rajinikanth V, et al. Firefly-algorithm supported scheme to detect COVID-19 lesion in lung ct scan images using Shannon entropy and Markov-random-field. arXiv preprint arXiv: 2004.09239. 2020.

124. Filipczuk P, Weronika W, Andrzej O. Automatic nuclei detection on cytological images using the firefly optimization algorithm. In: Information technologies in biomedicine. Springer, Berlin, Heidelberg; 2012. pp. 85-92.

125. Gadekallu TR, et al. Early detection of diabetic retinopathy using PCA-firefly based deep learning model. Electronics. 2020;9.2:274. 
126. Tuba E, Milan T, Dana S. Support vector machine optimized by firefly algorithm for emphysema classification in lung tissue CT images. http: //hdl.handle.net/11025/29747. 2017.

127. Malathi K, Nedunchelian R. Detecting and classifying diabetic retinopathy in fundus retina images using artificial neural networks-based firefly clustering algorithm. ARPN J Eng Appl Sci. 2016;11(5):3419-26.

128. Sivaprakash A, Samuel NER, Selvaperumal S. A novel robust medical image watermarking employing firefly optimization for secured telemedicine. J Med Imaging Health Inf. 2019;9.7:1373-1381.

129. Rani M, Laxmi P, Gottapu SR, Rao BP. An efficient codebook generation using firefly algorithm for optimum medical image compression. J Ambient Intell Human Comput. 2020;2020:1-13.

130. Chao C-F, Horng M-H, Chen Y-C (2015) Motion estimation using the firefly algorithm in ultrasonic image sequence of soft tissue. Comput Math Methods Med. https://doi. org $/ 10.1155 / 2015 / 343217$.

131. Dash S, Ajith A. Kernel based chaotic firefly algorithm for diagnosing Parkinson's disease. In: International conference on hybrid intelligent systems. Springer, Cham. 2018.

132. Keerthana K, et al. Retinal vessel extraction based on firefly algorithm guided multi-scale matched filter. Int J Modern Sci Technol. 2017;2(2):74-80.

133. Pereira Carla, Gonçalves Luís, Ferreira Manuel. Optic disc detection in color fundus images using ant colony optimization. Med Biol Eng Comput. 2013;51(3):295-303.

134. Deshpande MS, Pooja S, Honade SJ. (2017) Brain tumor segmentation and detection using firefly algorithm. J Electron Commun Eng. 2017;12(2):III.

135. Ahmed CRM, et al. To detect and classify oral cancer in mri image using firefly algorithm and expectation maximization algorithm. Int J Pure Appl Math. 2017;116.21:149-154.

136. Thawkar S, Ranjana I. Classification of masses in digital mammograms using firefly based optimization. Int J Image Graph Signal Process. 2018;10:2.

137. Srikanth MV, Prasad VVKDV, Satya Prasad K. An improved firefly algorithm-based 2-d image thresholding for brain image fusion. Int J Cognit Inf Natural Intell (IJCINI). 2020;14.3:60-96.

138. Sasikala S, Ezhilarasi M, Arun Kumar S. Detection of breast cancer using fusion of MLO and CC view features through a hybrid technique based on binary firefly algorithm and optimum-path forest classifier. Appl Nature-Inspired Comput Algor Case Stud Springer Singap. 2020;2020:23-40.

139. Liu Y, Ma L, Zhang Y. Thyroid extraction based on artificial immune network with firefly algorithm in single photon emission computed tomography image. J Med Imaging Health Inf. 2020;10(3):614-9.

140. Ghosh P, Sitansu KD, Kalyani M. "Comparative analysis of proposed FCM clustering integrated enhanced firefly-optimized algorithm (En-FAOFCM) for MR image segmentation and performance evaluation. J Image Process Pattern Recogn Progress. 2016;3.1:32-44.

141. Kumar A, Ansari MA, Alaknanda A. Brain tumor classification with optimized features using firefly algorithm. Indian J Public Health Res Dev. 2019;10:12.

142. Al-Thanoon NA, Omar SQ, Zakariya YA. Tuning parameter estimation in SCAD-support vector machine using firefly algorithm with application in gene selection and cancer classification. Comput Biol Med. 2018;103:262-268.

143. Karaboga D, Basturk B. A powerful and efficient algorithm for numerical function optimization: artificial bee colony (ABC) algorithm. J Global Optim. 2007;39(3):459-71.

144. Senapati MR, Pradipta KD. Local linear wavelet neural network based breast tumor classification using firefly algorithm. Neural Comput Appl. 2018;22.7-8:1591-1598.
145. Guijarro-Berdiñas B, et al. A linear learning method for multilayer perceptrons using least-squares. In: International conference on intelligent data engineering and automated learning. Springer, Berlin, Heidelberg; 2007.

146. Abonyi J, Szeifert F. Supervised fuzzy clustering for the identification of fuzzy classifiers. Pattern Recognit Lett. 2003;24(14):2195-207.

147. Hashem M, Hassanein AS (2018) Jaw fracture classification using meta heuristic firefly algorithm with multi-layered associative neural networks. Cluster Comput. https://doi.org/10.1007/ s10586-018-2668-z.

148. https://www.ncbi.nlm.nih.gov/projects/gap/cgibin/datas et.cgi?study_id=phs000007.v18.p7\&pht=1891. Accessed 3 Aug 2020.

149. Long NC, Phayung M, Herwig U. A highly accurate firefly based algorithm for heart disease prediction. Expert Syst Appl. 2015;42.21:8221-8231.

150. Sudheer C, et al. A support vector machine-firefly algorithm based forecasting model to determine malaria transmission. Neurocomputing. 2014;129:279-288.

151. Rajaguru H, Sunil KP. A study on firefly algorithm for breast cancer classification. In: International conference on ISMAC in computational vision and bio-engineering. Springer, Cham; 2018.

152. Umamaheswari TS, Sumathi P. Enhanced firefly algorithm (EFA) based gene selection and adaptive neuro neutrosophic inference system (ANNIS) prediction model for detection of circulating tumor cells (CTCs) in breast cancer analysis. Cluster Comput. 2018;2018:1-13.

153. Cheng M-Y, Hoang N-D. Estimating construction duration of diaphragm wall using firefly-tuned least squares support vector machine. Neural Comput Appl. 2018;30(8):2489-97.

154. Parveen SS, Kavitha C. Segmentation of CT lung nodules using FCM with firefly search algorithm. In: 2015 international conference on innovations in information, embedded and communication systems (ICIIECS). IEEE; 2015.

155. Mülayim N, Ayşegül A. Designing of an expert system based on firefly algorithm for diagnosis of Heart Disease. In: 2016 20th national biomedical engineering meeting (BIYOMUT). IEEE; 2016.

156. Ayas $\mathrm{S}$, et al. Microscopic image segmentation based on firefly algorithm for detection of tuberculosis bacteria. In: 2015 23nd signal processing and communications applications conference (SIU). IEEE, 2015.

157. Sánchez D, et al. A firefly algorithm for modular granular neural networks optimization applied to iris recognition. In: 2016 International Joint Conference on Neural Networks (IJCNN). IEEE; 2016.

158. Sadeghzadeh M. A new method for diagnosing breast cancer using firefly algorithm and fuzzy rule based classification. In: 2017 IEEE 11th international conference on application of information and communication technologies (AICT). IEEE; 2017.

159. Almugren N, Hala A. FF-SVM: new firefly-based gene selection algorithm for microarray cancer classification. In: 2019 IEEE conference on computational intelligence in bioinformatics and computational biology (CIBCB). IEEE; 2019.

160. Dash S, Ruppa T, Parimala T. An enhanced chaos-based firefly model for Parkinson's disease diagnosis and classification. In: 2017 international conference on information technology (ICIT). IEEE;; 2017.

161. Joyce PB, Rajinikanth V. Improving segmentation accuracy in biopsy cancer cell images using Otsu and Firefly Algorithm. Int J Appl Eng Res. 2014;9.24:8502-8506.

162. Rajakumar BR, Aloysius G. On hybridizing fuzzy min max neural network and firefly algorithm for automated heart disease diagnosis. In: 2013 Fourth international conference on 
computing, communications and networking technologies (ICCCNT). IEEE; 2013.

163. Yang X-S. A new metaheuristic bat-inspired algorithm. Nature inspired cooperative strategies for optimization (NICSO 2010). Springer, Berlin, Heidelberg, 2010. pp. 65-74
164. Nayak J, Bighnaraj N, Behera HS. A novel nature inspired firefly algorithm with higher order neural network: performance analysis. Eng Sci Technol Int J. 2016;19.1:197-211.

Publisher's Note Springer Nature remains neutral with regard to jurisdictional claims in published maps and institutional affiliations. 